\title{
STUDIES ON SORPTION PROPERTIES OF SOME BIO-POLYMERS IN THE ABSORPTION OF TOXIC METAL ION
}

\author{
Shreelatha Holla G \\ Department of Chemistry \\ S.C.E.M Adyar, Mangalore-7 \\ Karnataka, India
}

\author{
Dr. Savitha M.B \\ Department of Chemistry \\ S.C.E.M Adyar, Mangalore-7 \\ Karnataka, India
}

\author{
Dr. Prasad P \\ Department of Nanotechnology \\ S.I.T Valachil, Mangalore \\ Karnataka, India
}

\begin{abstract}
Toxic metal ions such as Fe (II) and Hg (II) present in industrial waste water can be absorbed by certain biopolymers in parts per milligrams. Biopolymers like Guar Gum, Xanthan Gum, Acasia Gum, Gum Tragacanth, Sodium Alginate, Carboxymethyl Cellulose, Methyl Cellulose and Hydroxypropyl Methyl Cellulose were tested for the same. We found $\mathrm{Hg}$ (II) metal ion can be absorbed only by Xanthan Gum and Gum Tragacanth whereas Fe (II) metal ion absorbed by all these biopolymers from waste water. Maximum possible absorption of metal ions determined using minimal quantity of bio-polymer sample. Sorption kinetics and solution property studies of biopolymers conducted also sorption of $\mathrm{Fe}, \mathrm{Hg}$ metal ions characterized at different temperature and concentrations of biopolymers. Absorption maximum for all these mixtures obtained through spectrophotometric study.
\end{abstract}

KEYWORDS: Absorption, Biopolymers, Sorption Property, Temperature effect.

\section{INTRODUCTION}

Sorption is a physical and chemical process whereas Biosorption is a physico-chemical process that occurs naturally. In these days treating industrial waste water is a challenge since it contains toxic metal ions, pesticides and other organic compounds. Certain existing methods for the treatment of industrial waste water are expensive and ineffective. So we can go for biosorption method which is environment friendly filtering technique. Biopolymers are produced by living organisms also they are renewable, compostable and biodegradable [10]. In order to remove these metal ions, waste water can be treated with some biopolymers which have hydrogel properties. Hydrogels are crosslinked polymers, hydrophilic in nature, able to swell in water $[1,4,9]$. Heavy metals are naturally occurring but various activities of human have altered the balance of the same. Once it enters into the environment or to the aquatic system heavy metals cannot be degraded or destroyed easily since they are stable. They tend to accumulate in nature [3]. They move from one ecological trophic level to another by damaging the ecosystem. Due to biomagnifications or multiplication process they accumulate in living tissues, causing several health issues in humans [12]. Many metallic elements like Zinc, Iron, Copper, etc. are essential for living organisms. Although they are necessary, they become toxic at high concentrations [11]. When present above threshold concentrations, all heavy metals can be toxic. Geological weathering, solid waste dump, leaching of metals, industrial processing of metals are some sources through which metal pollution occurs. Metals used in electroplating, tanning and textile industry are highly toxic to humans [6]. Existing biosorption methods for removal of metal ions from waste water like ion-exchange, reduction, precipitation, etc. are expensive and inefficient in treating large quantities. New trends in removing heavy metals from industrial waste water are being processed [2]. The natural affinity of biological compounds for metallic elements could contribute to the purification of metal loaded waste water [8]. Thus biosorption is a beneficial option because it is both efficient and cheap [5].

\section{EXPERIMENTAL}

\subsection{Materials and Methods}

Biopolymers selected based on availability and price. Guar gum (GG), Xanthan gum (XG), Acacia gum (Ac.G), Gum tragacanth (GT), Sodium alginate ( $\mathrm{Na} \mathrm{Alg}$ ), Carboxymethyl Cellulose (CMC), Methyl Cellulose (MC), Hydroxypropyl methyl cellulose (HPMC) were obtained from Himedia laboratories, Mumbai, India and used without additional purification. All chemicals were of Analytical reagent grade. Mohr's salt, $\mathrm{K}_{2} \mathrm{Cr}_{2} \mathrm{O}_{7}$ and $\mathrm{ZnSO}_{4} .7 \mathrm{H}_{2} \mathrm{O}$ obtained from Merck India, Ammonium thiocyanate obtained from Himedia. BaDS (Barium diphenylamine Sulfonate) indicator, $\mathrm{HgSO}_{4}$, Dithizone (1, 5-Diphenylthiocarbazone) and 1, 4-dioxane solution obtained from Loba chemie. Distilled water used wherever necessary. Digital $\mathrm{pH}$ meter (Systronics, MK-IV) used for $\mathrm{pH}$ study. Ultrasonic Interferometer for liquids F-81 (Mittal Enterprises) used for the estimation of ultrasonic sound velocity. Spectrophotometric analysis performed using Vis Double Beam Spectro 1203, Systronics. 


\section{International Journal of Engineering Applied Sciences and Technology, 2019 \\ Vol. 4, Issue 5, ISSN No. 2455-2143, Pages 285-301 \\ Published Online September 2019 in IJEAST (http://www.ijeast.com)}

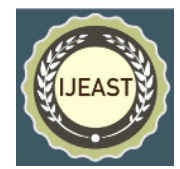

\subsection{Polymer sample preparation}

1000ppm Stock solution of $\mathrm{Fe}$ and $\mathrm{Hg}$ metal ions were prepared by Mohr's salt (Ferrous Ammonium Sulphate) and $\mathrm{HgSO}_{4}$ respectively. Crystals of Mohr's salt and $\mathrm{HgSO}_{4}$ were dissolved with dil. $\mathrm{H}_{2} \mathrm{SO}_{4}$. Bio-polymers such as Guar gum, Xanthan Gum, Acasia gum, Gum tragacanth, Sodium Alginate, CMC, Methyl cellulose and HPMC are taken with different quantities that are $20 \%(200 \mathrm{mg}), 15 \%(150 \mathrm{mg}), 10 \%(100 \mathrm{mg})$, $5 \%(50 \mathrm{mg}), 1 \%(10 \mathrm{mg}), 0.5 \%(5 \mathrm{mg}), 0.1 \%(1 \mathrm{mg}), 0.05 \%(0.5 \mathrm{mg})$ and $0.01 \%(0.1 \mathrm{mg})$. Each bio-polymer mixtures taken in different beakers, to that separately added $10 \mathrm{ml}$ of $1000 \mathrm{ppm} \mathrm{Fe}$ (II) and $\mathrm{Hg}$ (II) metal ion solution. Then for all the mixtures $10 \mathrm{ml}$ of waste water was added. These biopolymer- metal ion mixtures along with waste water mixed thoroughly with the help of magnetic stirrer. After proper mixing, the resulting mixture taken for further studies.

\subsection{Sample preparation for Spectrophotometric study}

For all biopolymer mixtures containing $\mathrm{Fe}$ (II) metal ion, ammonium thiocyanate solution and $\mathrm{HNO}_{3}$ were added. Whereas to the mixtures containing $\mathrm{Hg}$ (II) metal ion, equal volumes of Dithizone and $4.5 \mathrm{M}$ sulphuric acid were added. To this 1, 4-dioxane solution was added later.

\section{RESULTS AND DISCUSSIONS}

3.1 Interaction of bio-polymer mixtures with toxic metal ions

3.1.1 Study of absorption of Fe and $\mathrm{Hg}$ metal ions

Initially we applied simple titration method to determine the absorption properties of bio-polymers. Solution containing $10 \mathrm{ml}$ of $1000 \mathrm{ppm} \mathrm{Fe}$ (II) metal ion was titrated against $0.003 \mathrm{~N}$ $\mathrm{K}_{2} \mathrm{Cr}_{2} \mathrm{O}_{7}$ solution using $\mathrm{BaDS}$ (Barium diphenylamine Sulfonate) indicator at lab temperature $\left(30^{\circ} \mathrm{C}\right) . \quad \mathrm{K}_{2} \mathrm{Cr}_{2} \mathrm{O}_{7}$ standardised with $0.003 \mathrm{~N}$ Hypo solution. Solution containing $10 \mathrm{ml}$ of $1000 \mathrm{ppm} \mathrm{Hg}$ (II) metal ion was titrated against $0.02 \mathrm{M} \mathrm{ZnSO}_{4} .7 \mathrm{H}_{2} \mathrm{O}$ solution using EBT indicator at lab temperature $\left(30^{\circ} \mathrm{C}\right)$. Initially $\mathrm{ZnSO}_{4} .7 \mathrm{H}_{2} \mathrm{O}$ standardized with 0.02M EDTA. Strength of Mohr's salt ( $\mathrm{N}_{\text {Mohr's }}$ ) and Strength of $\mathrm{HgSO}_{4}\left(\mathrm{~N}_{\mathrm{HgSO}}\right)$ calculated using formula

$$
\mathrm{N}_{1} \mathrm{~V}_{1}=\mathrm{N}_{2} \mathrm{~V}_{2}
$$

Where $\mathrm{N}_{1}$ is the strength and $\mathrm{V}_{1}$ is volume of Mohr's salt solution, $\mathrm{N}_{2}$ is the strength and $\mathrm{V}_{2}$ is volume of $\mathrm{K}_{2} \mathrm{Cr}_{2} \mathrm{O}_{7}$ solution in case of $\mathrm{Fe}$ (II) analysis and $\mathrm{N}_{1}$ is the strength and $\mathrm{V}_{1}$ is volume of $\mathrm{HgSO}_{4}$ solution, $\mathrm{N}_{2}$ is the strength and $\mathrm{V}_{2}$ is volume of $\mathrm{ZnSO}_{4} .7 \mathrm{H}_{2} \mathrm{O}$ solution in case of $\mathrm{Hg}$ (II) analysis. By this, amount of only $\mathrm{Fe}$ and $\mathrm{Hg}$ can be calculated as

\section{$\mathrm{N}_{\text {Mohr's }}{ }^{*}$ Molar weight of $\mathrm{Fe}^{*} 1000$ in ppm.}

$\mathrm{N}_{\mathrm{HgSO} 4} *$ Molar weight of $\mathrm{Hg} * 1000$ in ppm.

Where $\mathrm{N}_{\text {Mohr's }}$ is the strength of Mohr's salt solution and $\mathrm{N}_{\mathrm{HgSO}}$ is the strength of $\mathrm{HgSO}_{4}$. Titration of $10 \mathrm{ml}$ of $1000 \mathrm{ppm}$ Mohr's salt solution against $0.003 \mathrm{~N} \mathrm{~K}_{2} \mathrm{Cr}_{2} \mathrm{O}_{7}$, considered as blank titration. Similarly titration of $10 \mathrm{ml}$ of $1000 \mathrm{ppm} \mathrm{HgSO}_{4}$ solution against $0.02 \mathrm{M} \mathrm{ZnSO} \mathrm{Zn}_{4} .7 \mathrm{H}_{2} \mathrm{O}$ solution considered as blank titration. Solution which contains only 1000ppm of Mohr's salt, considered as blank solution of $\mathrm{Fe}$ and solution contains only 1000ppm of $\mathrm{HgSO}_{4}$, considered as blank solution of $\mathrm{Hg}$. By titration method, amount of only $\mathrm{Fe}$ present in $1000 \mathrm{ppm}$ of Mohr's salt solution (blank solution of $\mathrm{Fe}$ ) was found to be $201 \mathrm{ppm}$ and amount of only $\mathrm{Hg}$ present in $1000 \mathrm{ppm}$ of $\mathrm{HgSO}_{4}$ solution (blank solution of $\mathrm{Hg}$ ) found to be $782 \mathrm{ppm}$ at lab temperature. Sorption properties of the metal ions are characterized by different concentrations of biopolymer mixtures. Then different concentrations of biopolymer mixtures along with corresponding metal ion solution were tested for absorption of $\mathrm{Fe}$ (II) and $\mathrm{Hg}$ (II) metal ions in the same way at lab temperature. It was observed that Guar gum, Xanthan gum, Acasia Gum, Gum tragacanth, Na Alginate, CMC, MC and HPMC mixtures could effectively absorb Fe (II) metal ion, only Xanthan gum and Gum tragacanth mixtures could absorb $\mathrm{Hg}$ (II) metal ion. Thus we can use corresponding bio-polymer mixtures for the effective removal of $\mathrm{Fe}$ (II) and $\mathrm{Hg}$ (II) metal ions from industrial waste water. Details of the observations have given in the Table 1.

Table 1. a.) Absorption of Fe (II) metal ion by different biopolymer mixtures $(\mathrm{ppm})$ at $30^{\circ} \mathrm{C}$.

\begin{tabular}{|c|c|c|c|c|c|c|c|c|c|}
\hline \multicolumn{10}{|c|}{ Amount of Fe (ppm) } \\
\hline $\begin{array}{l}\text { Concen } \\
\text { trations }\end{array}$ & $\begin{array}{l}20 \% \\
(200 \\
\mathrm{mg})\end{array}$ & $\begin{array}{l}15 \% \\
(150 \\
m g)\end{array}$ & $\begin{array}{l}10 \% \\
(100 \\
m g)\end{array}$ & $\begin{array}{l}5 \% \\
(50 \\
m g)\end{array}$ & $\begin{array}{l}1 \% \\
(10 \\
m g)\end{array}$ & $\begin{array}{c}0.5 \\
\% \\
(5 m \\
g)\end{array}$ & $\begin{array}{c}0.1 \\
\% \\
(1 \mathrm{~m} \\
\mathrm{g})\end{array}$ & $\begin{array}{c}0.05 \\
\% \\
(0.5 \\
\mathrm{mg})\end{array}$ & $\begin{array}{c}0.01 \\
\% \\
(0.1 \\
m g \\
\end{array}$ \\
\hline$G G$ & 114 & 121 & 129 & 134 & 137 & 140 & 144 & 147 & 151 \\
\hline$X G$ & 117 & 130 & 147 & 150 & 154 & 157 & 159 & 163 & 167 \\
\hline Ac. $G$ & 114 & 120 & 127 & 131 & 134 & 137 & 139 & 142 & 144 \\
\hline$G T$ & 107 & 116 & 122 & 128 & 134 & 140 & 144 & 146 & 149 \\
\hline Na Alg. & 134 & 137 & 141 & 145 & 147 & 150 & 154 & 157 & 159 \\
\hline CMC & 95 & 105 & 115 & 119 & 124 & 129 & 131 & 136 & 139 \\
\hline$M C$ & 117 & 126 & 134 & 136 & 139 & 141 & 144 & 146 & 149 \\
\hline НРМС & 119 & 128 & 139 & 143 & 149 & 152 & 156 & 159 & 162 \\
\hline
\end{tabular}

1. b.) Absorption of $\mathrm{Hg}$ (II) metal ion by $\mathrm{XG}$ and GT mixtures (ppm) at $30^{\circ} \mathrm{C}$.

\begin{tabular}{|c|c|c|c|c|c|c|c|c|c|}
\hline \multicolumn{10}{|c|}{ Amount of $\mathrm{Hg}$ (ppm) } \\
\hline $\begin{array}{c}\text { Conc } \\
\text { entrat } \\
\text { ions }\end{array}$ & $\begin{array}{l}20 \% \\
(200 \\
m g)\end{array}$ & $\begin{array}{l}15 \% \\
(150 \\
m g)\end{array}$ & $\begin{array}{l}10 \% \\
(100 \\
m g)\end{array}$ & $\begin{array}{l}5 \% \\
(50 \\
m g)\end{array}$ & $\begin{array}{l}1 \% \\
(10 \\
m g)\end{array}$ & $\begin{array}{c}0.5 \\
\% \\
(5 m \\
g) \\
\end{array}$ & $\begin{array}{c}0.1 \\
\% \\
(1 m \\
g) \\
\end{array}$ & $\begin{array}{c}0.05 \\
\% \\
(0.5 \\
m g) \\
\end{array}$ & $\begin{array}{c}0.01 \\
\% \\
(0.1 \\
m g) \\
\end{array}$ \\
\hline$X G$ & 538 & 572 & 610 & 644 & 678 & 690 & 702 & 715 & 722 \\
\hline$G T$ & 702 & 714 & 726 & 738 & 750 & 757 & 762 & 766 & 770 \\
\hline
\end{tabular}

At $30^{\circ} \mathrm{C}$ since the polymer concentration is high maximum absorption of $\mathrm{Fe}$ (II) and $\mathrm{Hg}$ (II) metal ions has observed for $20 \%$ each bio-polymer. According to the intension of this study we found that, minimal amount i.e., $0.01 \%(0.0001 \mathrm{gms})$ of biopolymer mixtures could absorb Fe metal ion as; $151 \mathrm{ppm}$ 


\section{International Journal of Engineering Applied Sciences and Technology, 2019 \\ Vol. 4, Issue 5, ISSN No. 2455-2143, Pages 285-301 \\ Published Online September 2019 in IJEAST (http://www.ijeast.com)}

using Guar gum, 167ppm using Xanthan gum, 144ppm using Acacia gum, 149ppm using GT, $159 \mathrm{ppm}$ using Sodium Alginate, $139 \mathrm{ppm}$ using CMC, $149 \mathrm{ppm}$ using MC and 162 ppm using HPMC mixture out of 201ppm (blank solution of Fe). Also $0.01 \%$ mixtures of XG and GT could absorb 722ppm of $\mathrm{Hg}$ metal ion and 770ppm of $\mathrm{Hg}$ metal ion respectively out of $782 \mathrm{ppm}$ (blank solution of $\mathrm{Hg}$ ) at lab temperature. The absorption of $\mathrm{Fe}$, $\mathrm{Hg}$ metal ions goes on increased as biopolymer concentrations decreased since concentration getting closer to blank concentration. i.e., $20 \%<15 \%<10 \%<5 \%<$ $1 \%<0.5 \%<0.1 \%<0.05 \%<0.01 \%$. Experiment has stopped at $20 \%(0.2 \mathrm{gms})$ of each bio-polymer mixture because it is difficult to carry out the experiment since the thickness of mixture becomes too high after $0.2 \mathrm{gms}$.

\subsection{2 pH study}

$\mathrm{pH}$ of blank $\mathrm{Fe}, \mathrm{Hg}$ solution and different concentrations of biopolymer mixtures were noted at lab temperature $\left(30^{\circ} \mathrm{C}\right)$. Acidic $\mathrm{pH}$ range of 2-3 has observed in blank solution of both $\mathrm{Fe}$ and $\mathrm{Hg}$ metal ion as well as in every bio-polymer mixtures with corresponding metal ion. $\mathrm{pH}$ of blank solution of $\mathrm{Fe}$ was found to be 3.81 and that of $\mathrm{Hg}$ was 3.10 at lab temperature. $\mathrm{pH}$ values of various biopolymer mixtures along with corresponding metal ion at lab temperature are mentioned in Table 2.

Table 2. a.) $\mathrm{pH}$ values of different biopolymer mixtures contain Fe (II) metal ion.

\begin{tabular}{|c|c|c|c|c|c|c|c|c|c|}
\hline $\begin{array}{c}\text { Co } \\
\text { nce } \\
\text { ntr } \\
\text { atio } \\
\text { ns }\end{array}$ & $\begin{array}{l}20 \% \\
(200 \\
m g)\end{array}$ & $\begin{array}{l}15 \% \\
(150 \\
m g)\end{array}$ & $\begin{array}{l}10 \% \\
(100 \\
m g)\end{array}$ & $\begin{array}{c}5 \% \\
(50 \\
m g)\end{array}$ & $\begin{array}{l}1 \% \\
(10 \\
m g)\end{array}$ & $\begin{array}{c}0.5 \\
\% \\
(5 m \\
g)\end{array}$ & $\begin{array}{c}0.1 \\
\% \\
(1 \mathrm{~lm} \\
\mathrm{g})\end{array}$ & $\begin{array}{c}0.05 \\
\% \\
(0.5 \\
\mathrm{mg})\end{array}$ & $\begin{array}{c}0.01 \\
\% \\
(0.1 \\
\mathrm{mg})\end{array}$ \\
\hline$G G$ & 3.78 & 3.63 & 3.85 & 3.80 & 3.74 & 3.71 & 3.80 & 3.83 & 3.81 \\
\hline$X G$ & 3.37 & 3.58 & 3.34 & 3.21 & 3.33 & 3.29 & 3.03 & 3.06 & 3.07 \\
\hline $\begin{array}{c}A c . \\
G\end{array}$ & 2.80 & 2.79 & 2.78 & 2.88 & 2.80 & 2.82 & 2.84 & 2.75 & 2.76 \\
\hline$G T$ & 2.87 & 2.85 & 2.88 & 2.89 & 2.78 & 2.80 & 2.88 & 2.79 & 2.89 \\
\hline $\begin{array}{l}\mathrm{Na} \\
\mathrm{Alg}\end{array}$ & 3.55 & 3.48 & 3.33 & 3.39 & 3.22 & 3.25 & 3.22 & 3.19 & 3.20 \\
\hline $\begin{array}{c}C M \\
C\end{array}$ & 3.33 & 3.17 & 3.10 & 3.07 & 2.94 & 3.00 & 2.91 & 2.96 & 2.90 \\
\hline$M C$ & 2.90 & 2.91 & 2.86 & 2.89 & 2.86 & 2.95 & 2.85 & 2.93 & 2.85 \\
\hline $\begin{array}{l}\boldsymbol{H P} \\
M C\end{array}$ & 3.15 & 3.12 & 3.15 & 3.16 & 3.11 & 3.10 & 3.12 & 3.15 & 3.10 \\
\hline
\end{tabular}

2. b.) $\mathrm{pH}$ values of $\mathrm{XG}$ and GT mixtures contain $\mathrm{Hg}$ (II) metal ion.

\begin{tabular}{|c|c|c|c|c|c|c|c|c|c|}
\hline $\begin{array}{c}\text { Co } \\
\text { nce } \\
\text { ntr } \\
\text { atio } \\
\text { ns }\end{array}$ & $\begin{array}{l}20 \% \\
(200 \\
m g)\end{array}$ & $\begin{array}{l}15 \% \\
(150 \\
\mathrm{mg})\end{array}$ & $\begin{array}{l}10 \% \\
(100 \\
m g)\end{array}$ & $\begin{array}{c}5 \% \\
(50 \\
m g)\end{array}$ & $\begin{array}{l}1 \% \\
(10 \\
m g)\end{array}$ & $\begin{array}{c}0.5 \\
\% \\
(5 m \\
g)\end{array}$ & $\begin{array}{c}0.1 \\
\% \\
(1 \mathrm{Im} \\
\mathrm{g})\end{array}$ & $\begin{array}{c}0.05 \\
\% \\
(0.5 \\
\mathrm{mg})\end{array}$ & $\begin{array}{c}0.01 \\
\% \\
(0.1 \\
m g)\end{array}$ \\
\hline$X G$ & 2.91 & 3.03 & 2.91 & 2.98 & 3.07 & 3.10 & 3.05 & 2.98 & 2.81 \\
\hline$G T$ & 3.08 & 3.07 & 3.10 & 2.79 & 2.98 & 2.88 & 2.94 & 3.08 & 2.82 \\
\hline
\end{tabular}

\subsubsection{Density and Viscosity studies of biopolymer mixture}

Density and viscosity measurements were carried out for both $\mathrm{Fe}$ and $\mathrm{Hg}$ blank solution and also for different concentrations of biopolymer mixtures with corresponding metal ions at lab temperature. At $30^{\circ} \mathrm{C}$ we found density of $\mathrm{Fe}$ blank was $0.3950 * 10^{3}\left(\mathrm{~kg} / \mathrm{m}^{3}\right)$ and its viscosity was $0.2805 \mathrm{~mm}^{2} / \mathrm{sec}$, density of $\mathrm{Hg}$ blank was $0.3950 * 10^{3}\left(\mathrm{~kg} / \mathrm{m}^{3}\right)$ and its viscosity was $0.3189 \mathrm{~mm}^{2} / \mathrm{sec}$. Density and viscosity values of various concentrations of biopolymer mixtures along with respective metal ions at are shown in Table 3 and 4 respectively. Both the parameters decreased as we move from higher concentration (20\%) to lower concentrations $(0.01 \%)$ of each biopolymer mixture. Mass of the substance goes on decreases from $20 \%$ to $0.01 \%$. As a result value of density decreases.

Thickness of the substance decreases from higher concentrations $(20 \%)$ to lower concentrations (0.01\%). Therefore internal resistance of the mixture decreases. We know that, a liquid with high internal resistance to flow is described as having high viscosity [7]. A liquid with low internal resistance to flow is described as having low viscosity. Therefore decrease in viscosity has observed from higher concentrations $(20 \%)$ to lower concentrations $(0.01 \%)$.

Table 3. a.) Density values of different biopolymer mixtures contain $\mathrm{Fe}$ (II) metal ion.

\begin{tabular}{|c|c|c|c|c|c|c|c|c|c|}
\hline \multirow[b]{2}{*}{$\begin{array}{c}\text { Co } \\
\text { nce } \\
\text { ntr } \\
\text { ati } \\
\text { ons }\end{array}$} & \multicolumn{9}{|c|}{ Density values $\left[10^{3}\left(\mathrm{~kg} / \mathrm{m}^{3}\right)\right]$ at $30^{\circ} \mathrm{C}$} \\
\hline & $\begin{array}{l}20 \% \\
(200 \\
m g)\end{array}$ & $\begin{array}{l}15 \% \\
(150 \\
m g)\end{array}$ & $\begin{array}{l}10 \% \\
(100 \\
m g)\end{array}$ & $\begin{array}{l}5 \% \\
(50 \\
m g)\end{array}$ & $\begin{array}{l}1 \% \\
(10 \\
m g)\end{array}$ & $\begin{array}{c}0.5 \\
\% \\
(5 m \\
g)\end{array}$ & $\begin{array}{c}0.1 \\
\% \\
(1 \mathrm{Im} \\
\mathrm{g})\end{array}$ & $\begin{array}{c}0.05 \\
\% \\
(0.5 \\
m g)\end{array}$ & $\begin{array}{c}0.01 \\
\% \\
(0.1 \\
m g)\end{array}$ \\
\hline $\boldsymbol{G G}$ & $\begin{array}{c}0.39 \\
70\end{array}$ & $\begin{array}{c}0.39 \\
69\end{array}$ & 68 & $\begin{array}{c}0.39 \\
67\end{array}$ & $\begin{array}{c}0.39 \\
66\end{array}$ & $\begin{array}{c}0.39 \\
65\end{array}$ & $\begin{array}{c}0.39 \\
64\end{array}$ & $\begin{array}{c}0.39 \\
63\end{array}$ & $\begin{array}{c}0.39 \\
61\end{array}$ \\
\hline$X G$ & $\begin{array}{c}0.397 \\
8\end{array}$ & $\begin{array}{c}0.39 \\
70\end{array}$ & $\begin{array}{c}0.39 \\
63\end{array}$ & $\begin{array}{c}0.39 \\
62\end{array}$ & $\begin{array}{c}0.39 \\
61\end{array}$ & $\begin{array}{c}0.39 \\
60\end{array}$ & $\begin{array}{c}0.39 \\
59\end{array}$ & $\begin{array}{c}0.39 \\
58\end{array}$ & $\begin{array}{c}0.39 \\
57\end{array}$ \\
\hline $\begin{array}{c}A c . \\
\boldsymbol{G}\end{array}$ & $\begin{array}{c}0.39 \\
75\end{array}$ & $\begin{array}{c}0.39 \\
73\end{array}$ & $\begin{array}{c}0.39 \\
69\end{array}$ & $\begin{array}{c}0.39 \\
65\end{array}$ & $\begin{array}{c}0.39 \\
61\end{array}$ & $\begin{array}{c}0.39 \\
59\end{array}$ & $\begin{array}{c}0.39 \\
57\end{array}$ & $\begin{array}{l}0.39 \\
55\end{array}$ & $\begin{array}{c}0.39 \\
54\end{array}$ \\
\hline$G T$ & $\begin{array}{c}0.39 \\
74\end{array}$ & $\begin{array}{c}0.39 \\
71\end{array}$ & $\begin{array}{c}0.39 \\
69\end{array}$ & $\begin{array}{c}0.39 \\
65\end{array}$ & $\begin{array}{c}0.39 \\
62\end{array}$ & $\begin{array}{c}0.39 \\
61\end{array}$ & $\begin{array}{c}0.39 \\
60\end{array}$ & $\begin{array}{l}0.39 \\
59\end{array}$ & $\begin{array}{c}0.39 \\
58\end{array}$ \\
\hline $\begin{array}{l}\mathrm{Na} \\
\mathrm{Alg}\end{array}$ & $\begin{array}{c}0.39 \\
80\end{array}$ & $\begin{array}{c}0.39 \\
74\end{array}$ & $\begin{array}{c}0.39 \\
69\end{array}$ & $\begin{array}{c}0.39 \\
67\end{array}$ & $\begin{array}{c}0.39 \\
66\end{array}$ & $\begin{array}{c}0.39 \\
64\end{array}$ & $\begin{array}{c}0.39 \\
62\end{array}$ & $\begin{array}{c}0.39 \\
61\end{array}$ & $\begin{array}{c}0.39 \\
60\end{array}$ \\
\hline $\begin{array}{c}C \\
M \\
C\end{array}$ & $\begin{array}{c}0.39 \\
73\end{array}$ & $\begin{array}{c}0.39 \\
71\end{array}$ & $\begin{array}{c}0.39 \\
68\end{array}$ & $\begin{array}{c}0.39 \\
65\end{array}$ & $\begin{array}{c}0.39 \\
63\end{array}$ & $\begin{array}{c}0.39 \\
62\end{array}$ & $\begin{array}{c}0.39 \\
61\end{array}$ & $\begin{array}{c}0.39 \\
58\end{array}$ & $\begin{array}{c}0.39 \\
55\end{array}$ \\
\hline $\begin{array}{c}M \\
C\end{array}$ & $\begin{array}{c}0.39 \\
71\end{array}$ & $\begin{array}{c}0.39 \\
66\end{array}$ & $\begin{array}{c}0.39 \\
62\end{array}$ & $\begin{array}{c}0.39 \\
61\end{array}$ & $\begin{array}{c}0.39 \\
60\end{array}$ & $\begin{array}{c}0.39 \\
59\end{array}$ & $\begin{array}{c}0.39 \\
58\end{array}$ & $\begin{array}{c}0.39 \\
57\end{array}$ & $\begin{array}{c}0.39 \\
56\end{array}$ \\
\hline $\begin{array}{c}H P \\
M \\
C\end{array}$ & $\begin{array}{c}0.39 \\
72\end{array}$ & $\begin{array}{c}0.39 \\
68\end{array}$ & $\begin{array}{c}0.39 \\
63\end{array}$ & $\begin{array}{c}0.39 \\
61\end{array}$ & $\begin{array}{c}0.39 \\
59\end{array}$ & $\begin{array}{c}0.39 \\
57\end{array}$ & $\begin{array}{c}0.39 \\
56\end{array}$ & $\begin{array}{c}0.39 \\
55\end{array}$ & $\begin{array}{c}0.39 \\
53\end{array}$ \\
\hline
\end{tabular}

3. b.) Density values of $X G$ and GT mixtures contain $\mathrm{Hg}$ (II) metal ion.

\begin{tabular}{c|c|c|c|c|c|c|c|c|c|}
\hline $\begin{array}{c}\text { Co } \\
\text { nce }\end{array}$ & \multicolumn{10}{|c|}{ Density values $\left[10^{3}\left(\mathrm{~kg}^{3}\right)\right]$ at $30^{\circ} \mathrm{C}$} \\
\cline { 2 - 10 } & $20 \%$ & $15 \%$ & $10 \%$ & $5 \%$ & $1 \%$ & 0.5 & 0.1 & 0.05 & 0.01 \\
ntr & $(200$ & $(150$ & $(100$ & $(50$ & $(10$ & $\%$ & $\%$ & $\%$ & $\%$ \\
\hline
\end{tabular}




\begin{tabular}{|c|c|c|c|c|c|c|c|c|c|}
\hline $\begin{array}{c}\text { atio } \\
\boldsymbol{n} \boldsymbol{s}\end{array}$ & $\boldsymbol{m g})$ & $\boldsymbol{m g})$ & $\boldsymbol{m g})$ & $\boldsymbol{m g})$ & $\boldsymbol{m g})$ & $\begin{array}{c}(\mathbf{5 m} \\
\boldsymbol{g})\end{array}$ & $\begin{array}{c}(\mathbf{m} \boldsymbol{g} \\
\boldsymbol{g})\end{array}$ & $\begin{array}{c}(\mathbf{0 . 5} \\
\mathbf{m g})\end{array}$ & $\begin{array}{c}(\mathbf{0 . 1} \\
\boldsymbol{m g})\end{array}$ \\
\hline $\boldsymbol{X} \boldsymbol{G}$ & $\begin{array}{c}0.40 \\
41\end{array}$ & $\begin{array}{c}0.40 \\
16\end{array}$ & $\begin{array}{c}0.40 \\
03\end{array}$ & $\begin{array}{c}0.39 \\
93\end{array}$ & $\begin{array}{c}0.39 \\
89\end{array}$ & $\begin{array}{c}0.39 \\
80\end{array}$ & $\begin{array}{c}0.39 \\
74\end{array}$ & $\begin{array}{c}0.39 \\
70\end{array}$ & $\begin{array}{c}0.39 \\
65\end{array}$ \\
\hline $\boldsymbol{G T}$ & $\begin{array}{c}0.39 \\
80\end{array}$ & $\begin{array}{c}0.39 \\
76\end{array}$ & $\begin{array}{c}0.39 \\
73\end{array}$ & $\begin{array}{c}0.39 \\
70\end{array}$ & $\begin{array}{c}0.39 \\
68\end{array}$ & $\begin{array}{c}0.39 \\
67\end{array}$ & $\begin{array}{c}0.39 \\
66\end{array}$ & $\begin{array}{c}0.39 \\
64\end{array}$ & $\begin{array}{c}0.39 \\
62\end{array}$ \\
\hline
\end{tabular}

Table 4. a.) Viscosity values of different biopolymer mixtures contain $\mathrm{Fe}$ (II) metal ion.

\begin{tabular}{|c|c|c|c|c|c|c|c|c|c|}
\hline Co & \multicolumn{9}{|c|}{ Viscosity values $\left(\mathrm{mm}^{2} / \mathrm{sec}\right)$ at $30^{\circ} \mathrm{C}$} \\
\hline $\begin{array}{c}\text { nce } \\
\text { ntr } \\
\text { ati } \\
\text { ons }\end{array}$ & $\begin{array}{l}20 \% \\
(200 \\
m g)\end{array}$ & $\begin{array}{l}15 \% \\
(150 \\
m g)\end{array}$ & $\begin{array}{l}10 \% \\
(100 \\
m g)\end{array}$ & $\begin{array}{c}5 \% \\
(50 \\
m g)\end{array}$ & $\begin{array}{l}1 \% \\
(10 \\
m g)\end{array}$ & $\begin{array}{c}0.5 \\
\% \\
(5 m \\
g) \\
\end{array}$ & $\begin{array}{c}0.1 \\
\% \\
(1 \mathrm{~m} \\
\mathrm{g}) \\
\end{array}$ & $\begin{array}{c}0.05 \\
\% \\
(0.5 \\
m g) \\
\end{array}$ & $\begin{array}{c}0.01 \\
\% \\
(0.1 \\
m g) \\
\end{array}$ \\
\hline$G G$ & $\begin{array}{l}14.4 \\
142\end{array}$ & $\begin{array}{c}9.98 \\
78\end{array}$ & $\begin{array}{c}4.80 \\
55\end{array}$ & $\begin{array}{c}2.18 \\
18\end{array}$ & $\begin{array}{c}0.69 \\
42\end{array}$ & $\begin{array}{c}0.56 \\
65\end{array}$ & $\begin{array}{c}0.34 \\
96\end{array}$ & $\begin{array}{c}0.32 \\
14\end{array}$ & $\begin{array}{c}0.29 \\
06\end{array}$ \\
\hline$X \boldsymbol{G}$ & $\begin{array}{l}36.6 \\
945\end{array}$ & $\begin{array}{c}25.4 \\
141\end{array}$ & $\begin{array}{l}13.8 \\
747\end{array}$ & $\begin{array}{c}8.31 \\
35\end{array}$ & $\begin{array}{c}2.32 \\
79\end{array}$ & $\begin{array}{c}1.17 \\
63\end{array}$ & $\begin{array}{c}0.89 \\
96\end{array}$ & $\begin{array}{c}0.50 \\
43\end{array}$ & $\begin{array}{c}0.29 \\
02\end{array}$ \\
\hline $\begin{array}{c}A c . \\
G\end{array}$ & $\begin{array}{c}0.43 \\
25\end{array}$ & $\begin{array}{c}0.41 \\
51\end{array}$ & $\begin{array}{c}0.40 \\
37\end{array}$ & $\begin{array}{c}0.39 \\
56\end{array}$ & $\begin{array}{c}0.37 \\
48\end{array}$ & $\begin{array}{c}0.36 \\
04\end{array}$ & $\begin{array}{c}0.34 \\
66\end{array}$ & $\begin{array}{c}0.32 \\
82\end{array}$ & $\begin{array}{c}0.31 \\
82\end{array}$ \\
\hline$G T$ & $\begin{array}{c}0.64 \\
86\end{array}$ & $\begin{array}{c}0.48 \\
48\end{array}$ & $\begin{array}{c}0.34 \\
73\end{array}$ & $\begin{array}{c}0.33 \\
65\end{array}$ & $\begin{array}{c}0.32 \\
80\end{array}$ & $\begin{array}{c}0.31 \\
61\end{array}$ & $\begin{array}{c}0.30 \\
91\end{array}$ & $\begin{array}{c}0.30 \\
03\end{array}$ & $\begin{array}{c}0.29 \\
49\end{array}$ \\
\hline $\begin{array}{l}\mathrm{Na} \\
\mathrm{Alg}\end{array}$ & $\begin{array}{c}0.41 \\
42\end{array}$ & $\begin{array}{c}0.40 \\
08\end{array}$ & $\begin{array}{c}0.38 \\
49\end{array}$ & $\begin{array}{c}0.36 \\
54\end{array}$ & $\begin{array}{c}0.34 \\
71\end{array}$ & $\begin{array}{c}0.33 \\
53\end{array}$ & $\begin{array}{c}0.32 \\
80\end{array}$ & $\begin{array}{c}0.31 \\
33\end{array}$ & $\begin{array}{c}0.30 \\
91\end{array}$ \\
\hline $\begin{array}{c}C \\
M \\
C\end{array}$ & $\begin{array}{c}2.25 \\
55\end{array}$ & $\begin{array}{c}1.86 \\
45\end{array}$ & $\begin{array}{c}0.76 \\
98\end{array}$ & $\begin{array}{c}0.55 \\
87\end{array}$ & $\begin{array}{c}0.38 \\
90\end{array}$ & $\begin{array}{c}0.35 \\
11\end{array}$ & $\begin{array}{c}0.32 \\
32\end{array}$ & $\begin{array}{c}0.31 \\
56\end{array}$ & $\begin{array}{c}0.30 \\
87\end{array}$ \\
\hline $\begin{array}{c}M \\
C\end{array}$ & $\begin{array}{c}7.61 \\
77\end{array}$ & $\begin{array}{c}5.67 \\
30\end{array}$ & $\begin{array}{c}1.59 \\
10\end{array}$ & $\begin{array}{c}0.77 \\
56\end{array}$ & $\begin{array}{c}0.35 \\
12\end{array}$ & $\begin{array}{c}0.32 \\
45\end{array}$ & $\begin{array}{c}0.29 \\
50\end{array}$ & $\begin{array}{c}0.29 \\
34\end{array}$ & $\begin{array}{c}0.29 \\
01\end{array}$ \\
\hline $\begin{array}{c}\boldsymbol{H P} \\
\boldsymbol{M} \\
\boldsymbol{C}\end{array}$ & $\begin{array}{c}1.21 \\
65\end{array}$ & $\begin{array}{c}0.99 \\
89\end{array}$ & $\begin{array}{c}0.56 \\
25\end{array}$ & $\begin{array}{c}0.44 \\
9\end{array}$ & $\begin{array}{c}0.30 \\
44\end{array}$ & $\begin{array}{c}0.29 \\
93\end{array}$ & 0.29 & $\begin{array}{c}0.28 \\
86\end{array}$ & $\begin{array}{c}0.28 \\
16\end{array}$ \\
\hline
\end{tabular}

4. b.) Viscosity values of $\mathrm{XG}$ and GT mixtures contain $\mathrm{Hg}$ (II) metal ion.

\begin{tabular}{|c|c|c|c|c|c|c|c|c|c|}
\hline \multirow[b]{2}{*}{$\begin{array}{c}\text { Co } \\
\text { nce } \\
\text { ntr } \\
\text { atio } \\
\text { ns }\end{array}$} & \multicolumn{9}{|c|}{ Viscosity values $\left(\mathrm{mm}^{2} / \mathrm{sec}\right)$ at $30^{\circ} \mathrm{C}$} \\
\hline & $\begin{array}{c}20 \% \\
(200 \\
m g)\end{array}$ & $\begin{array}{l}15 \% \\
(150 \\
\mathrm{mg})\end{array}$ & $\begin{array}{l}10 \% \\
(100 \\
m g)\end{array}$ & $\begin{array}{c}5 \% \\
(50 \\
m g)\end{array}$ & $\begin{array}{c}1 \% \\
(10 \\
m g)\end{array}$ & $\begin{array}{c}0.5 \\
\% \\
(5 m \\
g) \\
\end{array}$ & $\begin{array}{c}0.1 \\
\% \\
(1 m \\
g)\end{array}$ & $\begin{array}{c}0.05 \\
\% \\
(0.5 \\
\mathrm{mg}) \\
\end{array}$ & $\begin{array}{c}0.01 \\
\% \\
(0.1 \\
m g) \\
\end{array}$ \\
\hline$X G$ & $\begin{array}{c}28.4 \\
53\end{array}$ & $\begin{array}{c}17.3 \\
13\end{array}$ & 8.45 & $\begin{array}{c}4.06 \\
54\end{array}$ & $\begin{array}{c}2.34 \\
7\end{array}$ & $\begin{array}{c}0.76 \\
53\end{array}$ & $\begin{array}{c}0.43 \\
31\end{array}$ & $\begin{array}{c}0.38 \\
75\end{array}$ & $\begin{array}{c}0.32 \\
83\end{array}$ \\
\hline$G T$ & $\begin{array}{c}0.39 \\
07\end{array}$ & $\begin{array}{c}0.36 \\
4\end{array}$ & $\begin{array}{c}0.35 \\
71\end{array}$ & $\begin{array}{c}0.34 \\
21\end{array}$ & $\begin{array}{c}0.33 \\
32\end{array}$ & $\begin{array}{c}0.33 \\
08\end{array}$ & $\begin{array}{c}0.32 \\
84\end{array}$ & $\begin{array}{c}0.32 \\
08\end{array}$ & $\begin{array}{c}0.31 \\
99\end{array}$ \\
\hline
\end{tabular}

\subsubsection{Study of Ultrasonic sound velocity of biopolymer} mixtures

Determination of ultrasonic sound velocity carried out as a part of solution property studies of biopolymers with heavy metal ions. Study conducted for blank solution of $\mathrm{Fe}$ and $\mathrm{Hg}$ also for various concentrations of biopolymer mixture with corresponding metal ion at lab temperature using Ultrasonic Interferometer for liquids F-81 (Mittal Enterprises). Ultrasonic sound velocity of blank solution of $\mathrm{Fe}$ was $1609 \mathrm{~m} / \mathrm{s}$ and for blank $\mathrm{Hg}$ solution it was $1605 \mathrm{~m} / \mathrm{s}$. The obtained values of ultrasonic sound velocity for various concentrations of biopolymer mixtures with respective metal ions are given in Table 5. No characteristic changes observed in the values of ultrasonic velocity for different concentrations of biopolymer mixtures.

Table 5. a.) Ultrasonic sound velocities of different biopolymer mixtures contain $\mathrm{Fe}$ (II) metal ion.

\begin{tabular}{|c|c|c|c|c|c|c|c|c|c|}
\hline \multirow[b]{2}{*}{$\begin{array}{c}\text { Con } \\
\text { cent } \\
\text { ratio } \\
n\end{array}$} & \multicolumn{9}{|c|}{ Ultrasonic velocity $(\mathrm{m} / \mathrm{s})$ at $30^{\circ} \mathrm{C}$} \\
\hline & $\begin{array}{c}20 \% \\
(200 \\
m g)\end{array}$ & $\begin{array}{l}15 \% \\
(150 \\
m g)\end{array}$ & $\begin{array}{l}10 \% \\
(100 \\
m g)\end{array}$ & $\begin{array}{l}5 \% \\
(50 \\
m g)\end{array}$ & $\begin{array}{l}1 \% \\
(10 \\
m g)\end{array}$ & $\begin{array}{c}0.5 \\
\% \\
(5 m \\
g) \\
\end{array}$ & $\begin{array}{c}0.1 \\
\% \\
(1 m \\
g) \\
\end{array}$ & $\begin{array}{c}0.05 \\
\% \\
(0.5 \\
m g) \\
\end{array}$ & $\begin{array}{c}0.01 \\
\% \\
(0.1 \\
m g) \\
\end{array}$ \\
\hline$G G$ & 1724 & 1655 & 1646 & 1612 & 1708 & 1688 & 1630 & 1661 & 1645 \\
\hline$X G$ & 1609 & 1588 & 1607 & 1613 & 1594 & 1595 & 1604 & 1611 & 1616 \\
\hline $\begin{array}{c}A c . \\
\boldsymbol{G}\end{array}$ & 1575 & 1581 & 1565 & 1579 & 1590 & 1588 & 1566 & 1587 & 1597 \\
\hline$G T$ & 1621 & 1645 & 1630 & 1616 & 1636 & 1604 & 1639 & 1626 & 1620 \\
\hline $\begin{array}{c}\text { Na } \\
\text { Alg. }\end{array}$ & 1584 & 1596 & 1599 & 1579 & 1595 & 1575 & 1569 & 1591 & 1588 \\
\hline $\begin{array}{c}C M \\
C\end{array}$ & 1602 & 1598 & 1600 & 1621 & 1603 & 1597 & 1591 & 1616 & 1594 \\
\hline$M C$ & 1586 & 1593 & 1601 & 1603 & 1585 & 1590 & 1607 & 1587 & 1605 \\
\hline $\begin{array}{l}\boldsymbol{H P} \\
M C\end{array}$ & 1619 & 1624 & 1594 & 1612 & 1599 & 1587 & 1602 & 1579 & 1601 \\
\hline
\end{tabular}

5. b.) Ultrasonic sound velocities of $\mathrm{XG}$ and GT mixtures contain $\mathrm{Hg}$ (II) metal ion.

\begin{tabular}{|c|c|c|c|c|c|c|c|c|c|}
\hline \multirow[b]{2}{*}{$\begin{array}{c}\text { Con } \\
\text { cent } \\
\text { ratio } \\
\text { ns }\end{array}$} & \multicolumn{9}{|c|}{ Ultrasonic velocity $(\mathrm{m} / \mathrm{s})$ at $30^{\circ} \mathrm{C}$} \\
\hline & $\begin{array}{l}20 \% \\
(200 \\
m g)\end{array}$ & $\begin{array}{l}15 \% \\
(150 \\
m g)\end{array}$ & $\begin{array}{l}10 \% \\
(100 \\
\mathrm{mg})\end{array}$ & $\begin{array}{l}5 \% \\
(50 \\
m g)\end{array}$ & $\begin{array}{l}1 \% \\
(10 \\
m g)\end{array}$ & $\begin{array}{c}0.5 \\
\% \\
(5 m \\
g) \\
\end{array}$ & $\begin{array}{c}0.1 \\
\% \\
(1 \mathrm{~m} \\
\mathrm{g}) \\
\end{array}$ & $\begin{array}{c}0.05 \\
\% \\
(0.5 \\
m g) \\
\end{array}$ & $\begin{array}{c}0.01 \\
\% \\
(0.1 \\
m g) \\
\end{array}$ \\
\hline$X G$ & 1610 & 1595 & 1605 & 1607 & 1613 & 1616 & 1594 & 1610 & 1607 \\
\hline$G T$ & 1607 & 1604 & 1603 & 1600 & 1605 & 1608 & 1604 & 1602 & 1607 \\
\hline
\end{tabular}

\subsection{Sorption kinetics studies (Temperature Study)}

\subsubsection{Effect of temperature on absorption of toxic metal ion}

Study of effect of temperature on absorption of Fe (II) and $\mathrm{Hg}$ (II) by biopolymers, their $\mathrm{pH}$ values, density, viscosity and ultrasonic sound velocity were conducted. Both blank solution of $\mathrm{Fe}, \mathrm{Hg}$ and various concentrations of biopolymer mixtures containing corresponding metal ions were tested for the same. Temperature increased starting from lab temperature to slight higher temperatures. That was from $30^{\circ} \mathrm{C}$ to $60^{\circ} \mathrm{C}$. Absorption of $\mathrm{Fe}$ (II) and $\mathrm{Hg}$ (II) from industrial waste water was found to decrease with increasing temperature for all the biopolymer mixtures even for blank solutions of metal ion. As we increase the temperature the rate of reaction increases. Particles can 


\section{International Journal of Engineering Applied Sciences and Technology, 2019 \\ Vol. 4, Issue 5, ISSN No. 2455-2143, Pages 285-301 \\ Published Online September 2019 in IJEAST (http://www.ijeast.com)}

react only when they collide. By heating, particles move faster and collide more frequently. That will speed up the rate of reaction. Fig.1 and 2 represent the effect of temperature on absorption of $\mathrm{Fe}$ and $\mathrm{Hg}$ blank solutions respectively. Effect of temperature on absorption of Fe (II) metal ion by different concentrations of Guar gum mixtures (Fig.1.a), Xanthan gum mixtures (Fig.1.b), Acacia gum mixtures (Fig.1.c), Gum tragacanth mixtures (Fig.1.d), Sodium alginate mixtures (Fig.1.e), CMC mixtures (Fig.1.f), MC mixtures (Fig.1.g) and HPMC mixtures (Fig.1.h) have given below. Temperature effect on absorption of $\mathrm{Hg}$ (II) by Xanthan gum (Fig.2.a) and Gum tragacanth (Fig.2.b) also mentioned. Absorption curves of Fe, Hg blank (Fig.1 \& 2) taken as reference curve. Remaining curves of all biopolymer mixtures with corresponding metal ion when compared with respective reference curve, lesser absorption values obtained. From graph it is clear that temperature has greater effect on reaction rate of biopolymer mixtures resulting in, decreasing temperature curve. After $60^{\circ} \mathrm{C}$, absorption found to be constant also there was negligible difference in absorption values. Hence temperature study has stopped at $60^{\circ} \mathrm{C}$ for all the mixtures.

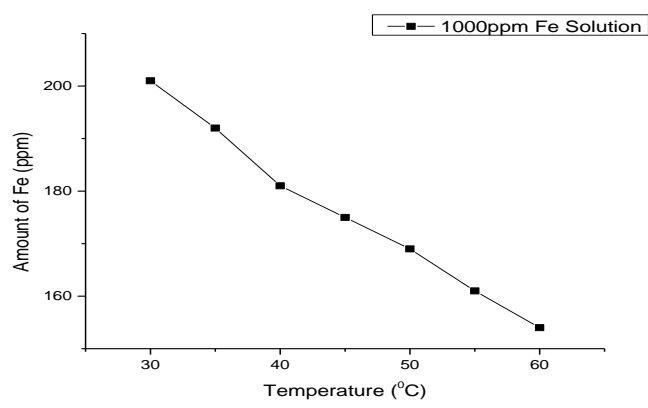

Fig.1 Effect of temperature on absorption of Fe blank

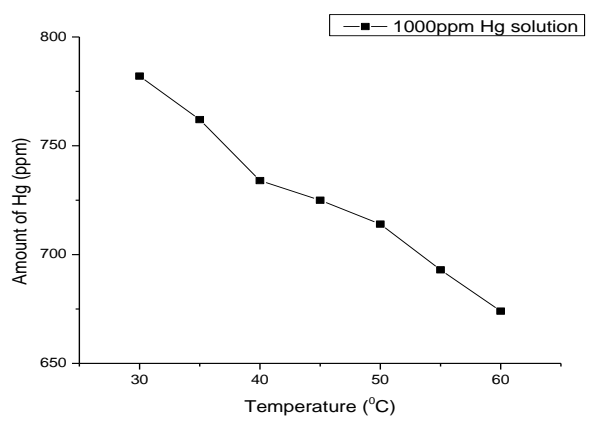

Fig.2 Effect of temperature on absorption of $\mathrm{Hg}$ blank

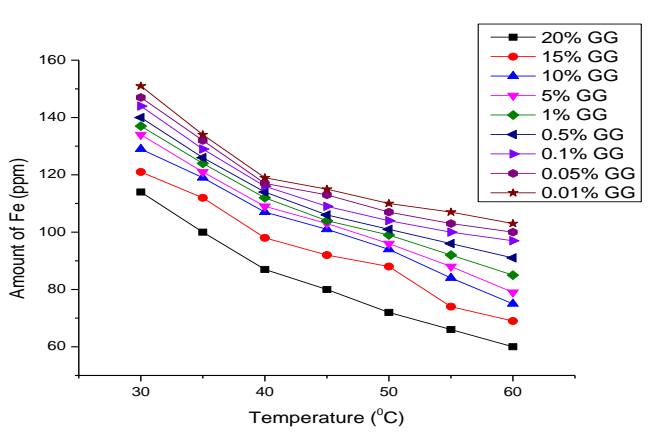

Fig.1.a Effect of temperature on absorption of Fe using GG mixtures

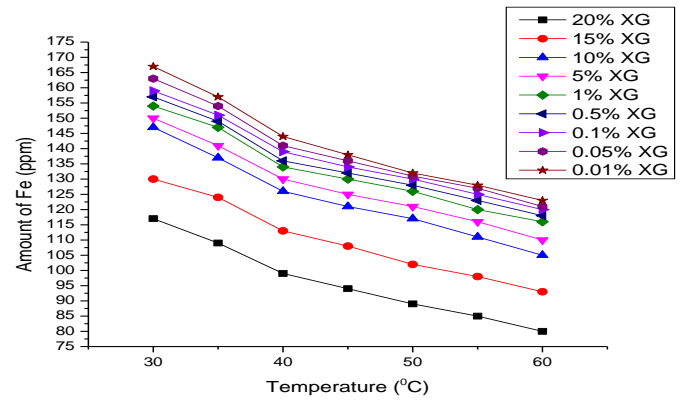

Fig.1.b Effect of temperature on absorption of Fe using XG mixtures

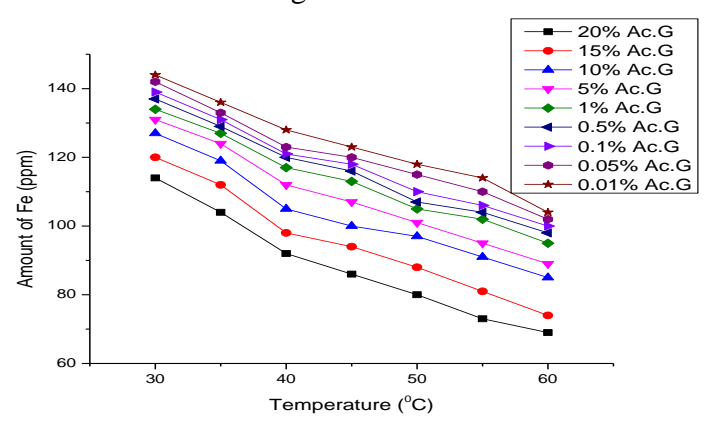

Fig.1.c Effect of temperature on absorption of Fe using Ac.G mixtures

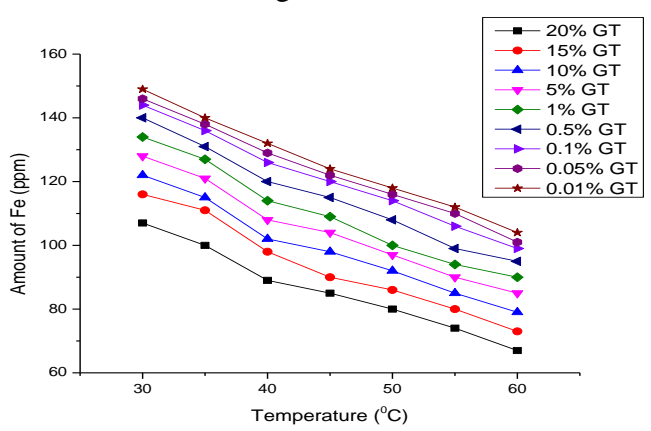

Fig.1.d Effect of temperature on absorption of Fe using GT mixtures. 


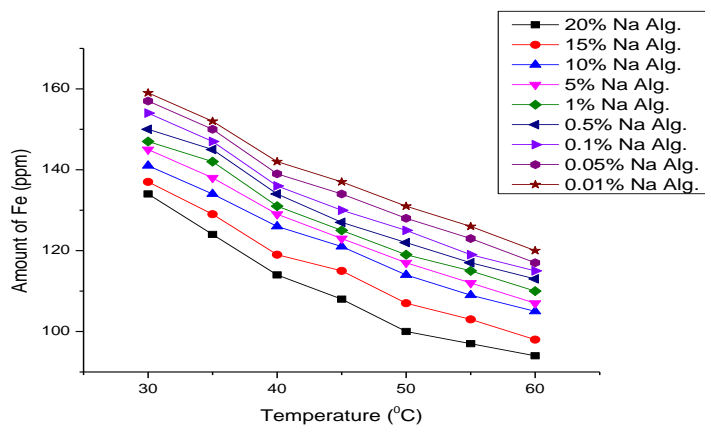

Fig.1.e Effect of temperature on absorption of Fe using $\mathrm{Na} \mathrm{Alg.} \mathrm{mixtures}$

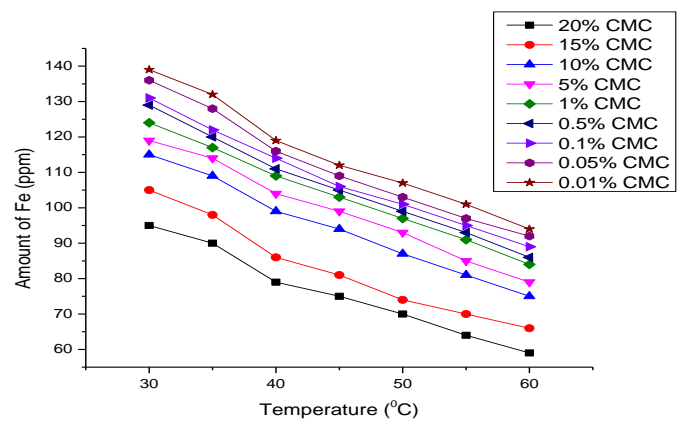

Fig.1.f Effect of temperature on absorption of Fe using CMC Mixtures

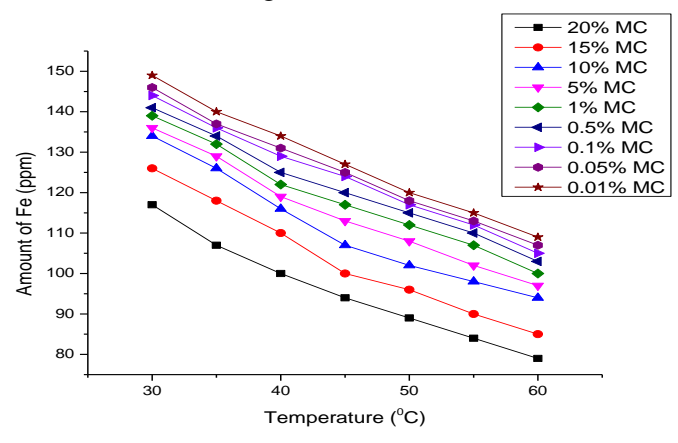

Fig.1.g Effect of temperature on absorption of Fe using MC mixtures

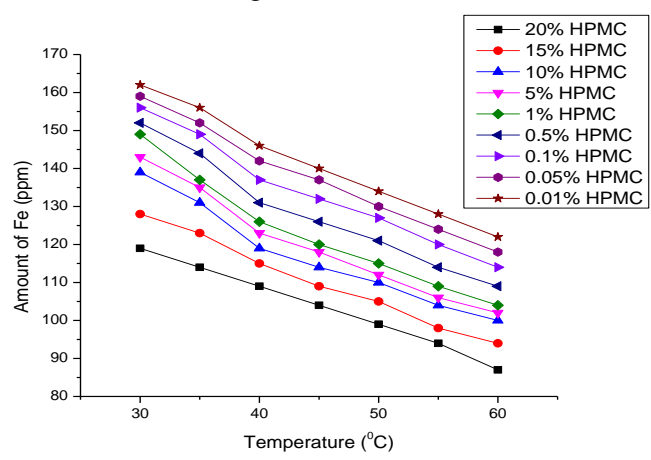

Fig.1.h Effect of temperature on absorption of Fe using HPMC mixtures

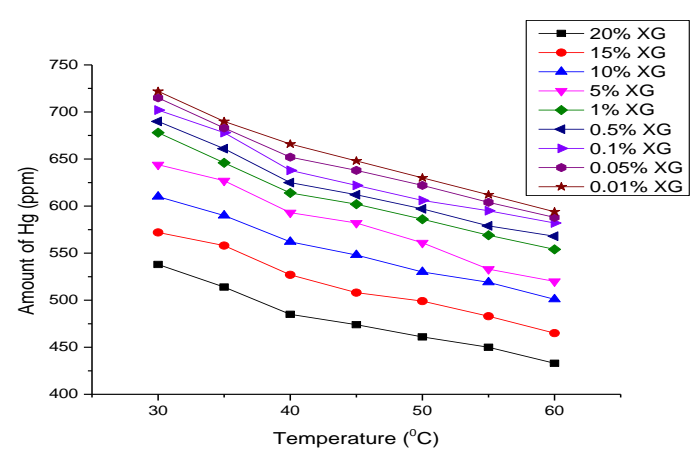

Fig.2.a Effect of temperature on absorption of $\mathrm{Hg}$ using XG mixtures

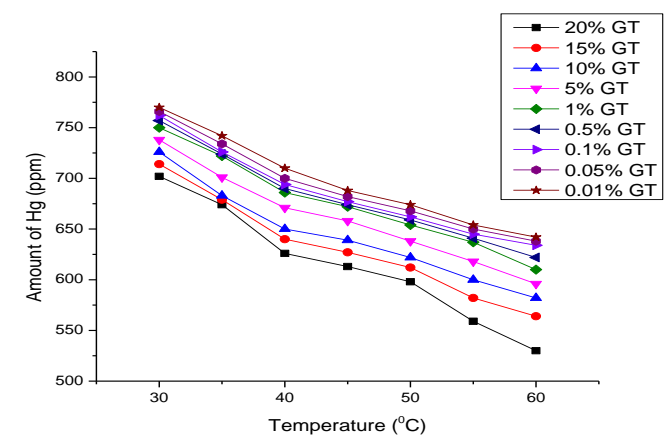

Fig.2.b Effect of temperature on absorption of $\mathrm{Hg}$ using GT Mixtures

\subsubsection{Effect of temperature on $\mathrm{pH}$ values}

$\mathrm{pH}$ of both blank solutions and their corresponding biopolymer mixtures were slightly decreased with increase in temperature. Acidic $\mathrm{pH}$ range $(2-3 \mathrm{pH})$ noticed in every mixture even though the $\mathrm{pH}$ values decreased. Temperature plays a significant role on $\mathrm{pH}$ measurements. As the temperature rises, molecular vibrations increase which results in the ability of water to ionize and form more hydrogen ions. As a result, the $\mathrm{pH}$ will drop. $\mathrm{pH}$ values of blank solutions of metal ion at different temperatures and effect of temperature on $\mathrm{pH}$ values of different concentrations of biopolymer mixtures are given in Table 6.

Table 6. a.) Effect of temperature on $\mathrm{pH}$ of $\mathrm{Fe}$ and $\mathrm{Hg}$ blank solutions

\begin{tabular}{|c|c|c|c|c|c|c|c|}
\hline $\begin{array}{c}\text { Tempe } \\
\text { rature } \\
{ }^{\circ} \boldsymbol{C}\end{array}$ & $\mathbf{3 0}$ & $\mathbf{3 5}$ & $\mathbf{4 0}$ & $\mathbf{4 5}$ & $\mathbf{5 0}$ & $\mathbf{5 5}$ & $\mathbf{6 0}$ \\
\hline $\begin{array}{c}\boldsymbol{p H} \text { of } \\
\boldsymbol{F e} \\
\text { blank }\end{array}$ & 3.81 & 3.66 & 3.14 & 3.06 & 2.98 & 2.88 & 2.68 \\
\hline $\begin{array}{c}\boldsymbol{p H} \text { of } \\
\mathbf{H g} \\
\text { blank }\end{array}$ & 3.10 & 3.06 & 2.96 & 2.86 & 2.83 & 2.79 & 2.77 \\
\hline
\end{tabular}


Published Online September 2019 in IJEAST (http://www.ijeast.com)

6. b.) Effect of temperature on $\mathrm{pH}$ of different biopolymer mixtures containing $\mathrm{Fe}$ metal ion

\begin{tabular}{|c|c|c|c|c|c|c|c|c|c|}
\hline \multirow{2}{*}{$\begin{array}{c}\text { Tem } \\
\text { pera } \\
\text { ture } \\
{ }^{\circ} \mathrm{C}\end{array}$} & \multicolumn{9}{|c|}{ GG mixtures } \\
\hline & $\begin{array}{l}20 \\
\%\end{array}$ & $\begin{array}{l}15 \\
\%\end{array}$ & $\begin{array}{l}10 \\
\%\end{array}$ & $5 \%$ & $1 \%$ & $\begin{array}{c}0.5 \\
\%\end{array}$ & $\begin{array}{l}0.1 \\
\%\end{array}$ & $\begin{array}{l}0.0 \\
5 \%\end{array}$ & $\begin{array}{c}0.01 \\
\%\end{array}$ \\
\hline 30 & $\begin{array}{c}3.7 \\
8\end{array}$ & $\begin{array}{c}3.6 \\
3\end{array}$ & $\begin{array}{c}3.8 \\
5\end{array}$ & $\begin{array}{c}3.8 \\
0\end{array}$ & $\begin{array}{c}3.7 \\
4\end{array}$ & $\begin{array}{c}3.7 \\
1\end{array}$ & $\begin{array}{c}3.8 \\
0\end{array}$ & $\begin{array}{c}3.8 \\
3\end{array}$ & 3.81 \\
\hline 35 & $\begin{array}{c}3.7 \\
0\end{array}$ & $\begin{array}{c}3.5 \\
0\end{array}$ & $\begin{array}{c}3.5 \\
5\end{array}$ & $\begin{array}{c}3.6 \\
3\end{array}$ & $\begin{array}{c}3.5 \\
3\end{array}$ & $\begin{array}{c}3.5 \\
9\end{array}$ & $\begin{array}{c}3.5 \\
8\end{array}$ & $\begin{array}{c}3.7 \\
6\end{array}$ & 3.49 \\
\hline 40 & $\begin{array}{c}3.6 \\
4\end{array}$ & $\begin{array}{c}3.2 \\
6\end{array}$ & $\begin{array}{c}3.0 \\
4\end{array}$ & $\begin{array}{c}3.4 \\
5\end{array}$ & $\begin{array}{c}3.1 \\
6\end{array}$ & $\begin{array}{c}3.4 \\
8\end{array}$ & $\begin{array}{c}3.1 \\
2\end{array}$ & $\begin{array}{c}3.5 \\
1\end{array}$ & 3.11 \\
\hline 45 & $\begin{array}{c}3.4 \\
6\end{array}$ & $\begin{array}{c}3.1 \\
1\end{array}$ & $\begin{array}{c}2.9 \\
8\end{array}$ & $\begin{array}{c}3.2 \\
9\end{array}$ & $\begin{array}{c}3.0 \\
4\end{array}$ & $\begin{array}{c}3.3 \\
1\end{array}$ & $\begin{array}{c}3.0 \\
1\end{array}$ & $\begin{array}{c}3.2 \\
8\end{array}$ & 3.02 \\
\hline 50 & $\begin{array}{c}3.0 \\
6\end{array}$ & $\begin{array}{c}2.9 \\
5\end{array}$ & $\begin{array}{c}2.9 \\
1\end{array}$ & $\begin{array}{c}3.1 \\
3\end{array}$ & $\begin{array}{c}2.8 \\
9\end{array}$ & $\begin{array}{c}3.1 \\
9\end{array}$ & $\begin{array}{c}2.9 \\
6\end{array}$ & $\begin{array}{c}3.1 \\
7\end{array}$ & 2.90 \\
\hline 55 & $\begin{array}{c}2.9 \\
4\end{array}$ & $\begin{array}{c}2.8 \\
8\end{array}$ & $\begin{array}{c}2.8 \\
8\end{array}$ & $\begin{array}{c}3.0 \\
1\end{array}$ & $\begin{array}{c}2.8 \\
6\end{array}$ & $\begin{array}{c}3.0 \\
6\end{array}$ & $\begin{array}{c}2.9 \\
1\end{array}$ & $\begin{array}{c}3.0 \\
9\end{array}$ & 2.84 \\
\hline 60 & $\begin{array}{c}2.8 \\
5\end{array}$ & $\begin{array}{c}2.7 \\
9\end{array}$ & $\begin{array}{c}2.8 \\
3\end{array}$ & 2.9 & $\begin{array}{c}2.8 \\
4\end{array}$ & $\begin{array}{c}2.8 \\
8\end{array}$ & $\begin{array}{c}2.8 \\
0\end{array}$ & $\begin{array}{c}2.8 \\
7\end{array}$ & 2.75 \\
\hline Tem & \multicolumn{9}{|c|}{$X G$ mixtures } \\
\hline $\begin{array}{c}\text { ture } \\
{ }^{0} \mathrm{C}\end{array}$ & $\begin{array}{l}20 \\
\%\end{array}$ & $\begin{array}{l}15 \\
\%\end{array}$ & $\begin{array}{l}10 \\
\%\end{array}$ & $5 \%$ & $1 \%$ & $\begin{array}{c}0.5 \\
\%\end{array}$ & $\begin{array}{c}0.1 \\
\%\end{array}$ & $\begin{array}{l}0.0 \\
5 \%\end{array}$ & $\begin{array}{c}0.01 \\
\%\end{array}$ \\
\hline 30 & $\begin{array}{c}3.3 \\
7\end{array}$ & $\begin{array}{c}3.5 \\
8\end{array}$ & $\begin{array}{c}3.3 \\
4\end{array}$ & $\begin{array}{c}3.2 \\
1\end{array}$ & $\begin{array}{c}3.3 \\
3\end{array}$ & $\begin{array}{c}3.2 \\
9\end{array}$ & $\begin{array}{c}3.0 \\
3\end{array}$ & $\begin{array}{c}3.0 \\
6\end{array}$ & 3.07 \\
\hline 35 & $\begin{array}{c}3.2 \\
8\end{array}$ & $\begin{array}{c}3.4 \\
4\end{array}$ & $\begin{array}{c}3.2 \\
5\end{array}$ & $\begin{array}{c}3.1 \\
4\end{array}$ & $\begin{array}{c}3.2 \\
8\end{array}$ & $\begin{array}{c}3.1 \\
8\end{array}$ & $\begin{array}{c}2.8 \\
8\end{array}$ & $\begin{array}{c}3.0 \\
2\end{array}$ & 2.98 \\
\hline 40 & $\begin{array}{c}3.0 \\
6\end{array}$ & $\begin{array}{c}3.3 \\
2\end{array}$ & $\begin{array}{c}3.0 \\
2\end{array}$ & $\begin{array}{c}3.0 \\
5\end{array}$ & $\begin{array}{c}3.0 \\
9\end{array}$ & $\begin{array}{c}3.1 \\
1\end{array}$ & $\begin{array}{c}2.8 \\
3\end{array}$ & $\begin{array}{c}2.9 \\
5\end{array}$ & 2.86 \\
\hline 45 & $\begin{array}{c}2.9 \\
9\end{array}$ & $\begin{array}{c}3.1 \\
6\end{array}$ & $\begin{array}{c}2.9 \\
5\end{array}$ & $\begin{array}{c}2.9 \\
6\end{array}$ & $\begin{array}{c}3.0 \\
1\end{array}$ & $\begin{array}{c}3.0 \\
4\end{array}$ & $\begin{array}{c}2.7 \\
9\end{array}$ & $\begin{array}{c}2.8 \\
8\end{array}$ & 2.79 \\
\hline 50 & $\begin{array}{c}2.9 \\
4\end{array}$ & $\begin{array}{c}3.0 \\
7\end{array}$ & $\begin{array}{c}2.8 \\
8\end{array}$ & $\begin{array}{c}2.8 \\
8\end{array}$ & $\begin{array}{c}2.9 \\
3\end{array}$ & $\begin{array}{c}2.9 \\
7\end{array}$ & $\begin{array}{c}2.7 \\
6\end{array}$ & $\begin{array}{c}2.8 \\
1\end{array}$ & 2.75 \\
\hline 55 & $\begin{array}{c}2.9 \\
0\end{array}$ & $\begin{array}{c}2.9 \\
7\end{array}$ & $\begin{array}{c}2.8 \\
1\end{array}$ & $\begin{array}{c}2.8 \\
1\end{array}$ & $\begin{array}{c}2.8 \\
5\end{array}$ & $\begin{array}{c}2.9 \\
0\end{array}$ & $\begin{array}{c}2.7 \\
1\end{array}$ & $\begin{array}{c}2.7 \\
4\end{array}$ & 2.73 \\
\hline 60 & $\begin{array}{c}2.8 \\
5\end{array}$ & $\begin{array}{c}2.8 \\
6\end{array}$ & $\begin{array}{c}2.7 \\
7\end{array}$ & $\begin{array}{c}2.7 \\
7\end{array}$ & $\begin{array}{c}2.7 \\
1\end{array}$ & $\begin{array}{c}2.8 \\
2\end{array}$ & $\begin{array}{c}2.6 \\
4\end{array}$ & $\begin{array}{c}2.7 \\
0\end{array}$ & 2.69 \\
\hline
\end{tabular}

\section{Ac.G mixtures}

\begin{tabular}{|c|c|c|c|c|c|c|c|c|c|}
\hline $\begin{array}{c}\text { Tem } \\
\text { pera } \\
\text { ture } \\
{ }^{\boldsymbol{O}} \boldsymbol{C}\end{array}$ & $\begin{array}{c}\mathbf{2 0} \\
\mathbf{\%}\end{array}$ & $\begin{array}{c}\mathbf{1 5} \\
\mathbf{\%}\end{array}$ & $\begin{array}{c}\mathbf{1 0} \\
\mathbf{\%}\end{array}$ & $\mathbf{5 \%}$ & $\mathbf{1 \%}$ & $\begin{array}{c}\mathbf{0 . 5} \\
\mathbf{\%}\end{array}$ & $\begin{array}{c}\mathbf{0 . 1} \\
\mathbf{\%}\end{array}$ & $\begin{array}{c}\mathbf{0 . 0} \\
\mathbf{5 \%}\end{array}$ & $\begin{array}{c}\mathbf{0 . 0 1} \\
\mathbf{\%}\end{array}$ \\
\hline $\mathbf{3 0}$ & $\begin{array}{c}2.8 \\
0\end{array}$ & $\begin{array}{c}2.7 \\
9\end{array}$ & $\begin{array}{c}2.7 \\
8\end{array}$ & $\begin{array}{c}2.8 \\
8\end{array}$ & $\begin{array}{c}2.8 \\
0\end{array}$ & $\begin{array}{c}2.8 \\
2\end{array}$ & $\begin{array}{c}2.8 \\
4\end{array}$ & $\begin{array}{c}2.7 \\
5\end{array}$ & 2.76 \\
\hline 35 & $\begin{array}{c}2.7 \\
4\end{array}$ & $\begin{array}{c}2.7 \\
3\end{array}$ & $\begin{array}{c}2.7 \\
5\end{array}$ & $\begin{array}{c}2.8 \\
1\end{array}$ & $\begin{array}{c}2.7 \\
7\end{array}$ & $\begin{array}{c}2.7 \\
6\end{array}$ & $\begin{array}{c}2.8 \\
0\end{array}$ & $\begin{array}{c}2.6 \\
9\end{array}$ & 2.73 \\
\hline $\mathbf{4 0}$ & $\begin{array}{c}2.7 \\
3\end{array}$ & $\begin{array}{c}2.6 \\
6\end{array}$ & $\begin{array}{c}2.7 \\
2\end{array}$ & $\begin{array}{c}2.7 \\
7\end{array}$ & $\begin{array}{c}2.7 \\
3\end{array}$ & $\begin{array}{c}2.7 \\
0\end{array}$ & $\begin{array}{c}2.7 \\
7\end{array}$ & $\begin{array}{c}2.6 \\
4\end{array}$ & 2.68 \\
\hline
\end{tabular}

\begin{tabular}{|c|c|c|c|c|c|c|c|c|c|}
\hline 45 & $\begin{array}{c}2.7 \\
1\end{array}$ & $\begin{array}{c}2.6 \\
4\end{array}$ & $\begin{array}{c}2.7 \\
0\end{array}$ & $\begin{array}{c}2.7 \\
4\end{array}$ & $\begin{array}{c}2.7 \\
1\end{array}$ & $\begin{array}{c}2.6 \\
7\end{array}$ & $\begin{array}{c}2.7 \\
6\end{array}$ & $\begin{array}{c}2.5 \\
8\end{array}$ & 2.67 \\
\hline 50 & $\begin{array}{c}2.7 \\
0\end{array}$ & $\begin{array}{c}2.5 \\
1\end{array}$ & $\begin{array}{c}2.6 \\
9\end{array}$ & $\begin{array}{c}2.6 \\
9\end{array}$ & $\begin{array}{c}2.7 \\
0\end{array}$ & $\begin{array}{c}2.6 \\
5\end{array}$ & $\begin{array}{c}2.7 \\
4\end{array}$ & $\begin{array}{c}2.4 \\
5\end{array}$ & 2.66 \\
\hline 55 & $\begin{array}{c}2.6 \\
8\end{array}$ & $\begin{array}{c}2.4 \\
1\end{array}$ & $\begin{array}{c}2.6 \\
5\end{array}$ & $\begin{array}{c}2.6 \\
3\end{array}$ & $\begin{array}{c}2.6 \\
8\end{array}$ & $\begin{array}{c}2.6 \\
3\end{array}$ & $\begin{array}{c}2.7 \\
1\end{array}$ & $\begin{array}{c}2.4 \\
2\end{array}$ & 2.63 \\
\hline 60 & $\begin{array}{c}2.6 \\
6\end{array}$ & $\begin{array}{c}2.3 \\
6\end{array}$ & $\begin{array}{c}2.6 \\
4\end{array}$ & $\begin{array}{c}2.5 \\
8\end{array}$ & $\begin{array}{c}2.6 \\
6\end{array}$ & $\begin{array}{c}2.5 \\
8\end{array}$ & $\begin{array}{c}2.6 \\
9\end{array}$ & $\begin{array}{c}2.3 \\
9\end{array}$ & 2.61 \\
\hline \multicolumn{10}{|c|}{ GT mixtures } \\
\hline $\begin{array}{c}\text { Tem } \\
\text { pera } \\
\text { ture } \\
{ }^{\circ} \mathrm{C}\end{array}$ & $\begin{array}{l}20 \\
\%\end{array}$ & $\begin{array}{l}15 \\
\%\end{array}$ & $\begin{array}{l}10 \\
\%\end{array}$ & $5 \%$ & $1 \%$ & $\begin{array}{c}0.5 \\
\%\end{array}$ & $\begin{array}{c}0.1 \\
\%\end{array}$ & $\begin{array}{l}0.0 \\
5 \%\end{array}$ & $\begin{array}{c}0.01 \\
\%\end{array}$ \\
\hline 30 & $\begin{array}{c}2.8 \\
7\end{array}$ & $\begin{array}{c}2.8 \\
5\end{array}$ & $\begin{array}{c}2.8 \\
8\end{array}$ & $\begin{array}{c}2.8 \\
9\end{array}$ & $\begin{array}{c}2.7 \\
8\end{array}$ & $\begin{array}{c}2.8 \\
0\end{array}$ & $\begin{array}{c}2.8 \\
8\end{array}$ & $\begin{array}{c}2.7 \\
9\end{array}$ & 2.89 \\
\hline 35 & $\begin{array}{c}2.8 \\
6\end{array}$ & $\begin{array}{c}2.8 \\
1\end{array}$ & $\begin{array}{c}2.8 \\
6\end{array}$ & $\begin{array}{c}2.8 \\
3\end{array}$ & $\begin{array}{c}2.7 \\
5\end{array}$ & $\begin{array}{c}2.7 \\
4\end{array}$ & $\begin{array}{c}2.8 \\
5\end{array}$ & $\begin{array}{c}2.7 \\
3\end{array}$ & 2.84 \\
\hline 40 & $\begin{array}{c}2.8 \\
4\end{array}$ & $\begin{array}{c}2.7 \\
4\end{array}$ & $\begin{array}{c}2.8 \\
2\end{array}$ & $\begin{array}{c}2.7 \\
2\end{array}$ & $\begin{array}{c}2.7 \\
0\end{array}$ & $\begin{array}{c}2.7 \\
0\end{array}$ & $\begin{array}{c}2.8 \\
1\end{array}$ & $\begin{array}{c}2.6 \\
6\end{array}$ & 2.81 \\
\hline 45 & $\begin{array}{c}2.8 \\
2\end{array}$ & $\begin{array}{c}2.7 \\
0\end{array}$ & $\begin{array}{c}2.8 \\
0\end{array}$ & $\begin{array}{c}2.6 \\
6\end{array}$ & $\begin{array}{c}2.6 \\
8\end{array}$ & $\begin{array}{c}2.6 \\
5\end{array}$ & $\begin{array}{c}2.8 \\
0\end{array}$ & $\begin{array}{c}2.5 \\
5\end{array}$ & 2.80 \\
\hline 50 & $\begin{array}{c}2.8 \\
0\end{array}$ & $\begin{array}{c}2.6 \\
6\end{array}$ & $\begin{array}{c}2.7 \\
8\end{array}$ & $\begin{array}{c}2.6 \\
3\end{array}$ & $\begin{array}{c}2.6 \\
6\end{array}$ & $\begin{array}{c}2.6 \\
1\end{array}$ & $\begin{array}{c}2.7 \\
7\end{array}$ & $\begin{array}{c}2.4 \\
8\end{array}$ & 2.78 \\
\hline 55 & $\begin{array}{c}2.7 \\
8\end{array}$ & $\begin{array}{c}2.5 \\
1\end{array}$ & $\begin{array}{c}2.7 \\
6\end{array}$ & $\begin{array}{c}2.5 \\
8\end{array}$ & $\begin{array}{c}2.6 \\
4\end{array}$ & $\begin{array}{c}2.5 \\
5\end{array}$ & $\begin{array}{c}2.7 \\
4\end{array}$ & $\begin{array}{c}2.4 \\
1\end{array}$ & 2.74 \\
\hline 60 & $\begin{array}{c}2.7 \\
6\end{array}$ & $\begin{array}{c}2.4 \\
6\end{array}$ & $\begin{array}{c}2.7 \\
4\end{array}$ & $\begin{array}{c}2.5 \\
0\end{array}$ & $\begin{array}{c}2.6 \\
1\end{array}$ & $\begin{array}{c}2.5 \\
0\end{array}$ & $\begin{array}{c}2.7 \\
2\end{array}$ & $\begin{array}{c}2.3 \\
2\end{array}$ & 2.72 \\
\hline \multicolumn{10}{|c|}{ Na Alg. Mixtures } \\
\hline $\begin{array}{c}\text { Tem } \\
\text { pera } \\
\text { ture } \\
{ }^{\circ} \mathrm{C}\end{array}$ & $\begin{array}{l}20 \\
\%\end{array}$ & $\begin{array}{l}15 \\
\%\end{array}$ & $\begin{array}{l}10 \\
\%\end{array}$ & $5 \%$ & $1 \%$ & $\begin{array}{c}0.5 \\
\%\end{array}$ & $\begin{array}{c}0.1 \\
\%\end{array}$ & $\begin{array}{l}0.0 \\
5 \%\end{array}$ & $\begin{array}{c}0.01 \\
\%\end{array}$ \\
\hline 30 & $\begin{array}{c}3.5 \\
5\end{array}$ & $\begin{array}{c}3.4 \\
8\end{array}$ & $\begin{array}{c}3.3 \\
3\end{array}$ & $\begin{array}{c}3.3 \\
9\end{array}$ & $\begin{array}{c}3.2 \\
2\end{array}$ & $\begin{array}{c}3.2 \\
5\end{array}$ & $\begin{array}{c}3.2 \\
2\end{array}$ & $\begin{array}{c}3.1 \\
9\end{array}$ & 3.20 \\
\hline 35 & $\begin{array}{c}3.4 \\
6\end{array}$ & $\begin{array}{c}3.3 \\
6\end{array}$ & $\begin{array}{c}3.2 \\
8\end{array}$ & $\begin{array}{c}3.3 \\
1\end{array}$ & $\begin{array}{c}3.1 \\
7\end{array}$ & $\begin{array}{c}3.2 \\
0\end{array}$ & $\begin{array}{c}3.1 \\
5\end{array}$ & $\begin{array}{c}3.1 \\
5\end{array}$ & 3.12 \\
\hline 40 & $\begin{array}{c}3.4 \\
2\end{array}$ & $\begin{array}{c}3.1 \\
8\end{array}$ & $\begin{array}{c}3.2 \\
2\end{array}$ & $\begin{array}{c}3.2 \\
5\end{array}$ & $\begin{array}{c}3.1 \\
2\end{array}$ & $\begin{array}{c}3.1 \\
5\end{array}$ & $\begin{array}{c}3.1 \\
1\end{array}$ & $\begin{array}{c}3.1 \\
1\end{array}$ & 3.05 \\
\hline 45 & $\begin{array}{c}3.3 \\
3\end{array}$ & $\begin{array}{c}3.1 \\
1\end{array}$ & $\begin{array}{c}3.2 \\
0\end{array}$ & $\begin{array}{c}3.1 \\
7\end{array}$ & $\begin{array}{c}3.0 \\
7\end{array}$ & $\begin{array}{c}3.1 \\
3\end{array}$ & $\begin{array}{c}3.0 \\
8\end{array}$ & $\begin{array}{c}3.0 \\
6\end{array}$ & 3.02 \\
\hline 50 & $\begin{array}{c}3.2 \\
9\end{array}$ & $\begin{array}{c}3.0 \\
5\end{array}$ & $\begin{array}{c}3.1 \\
8\end{array}$ & $\begin{array}{c}3.1 \\
1\end{array}$ & $\begin{array}{c}3.0 \\
5\end{array}$ & $\begin{array}{c}3.0 \\
6\end{array}$ & $\begin{array}{c}3.0 \\
6\end{array}$ & $\begin{array}{c}3.0 \\
4\end{array}$ & 2.98 \\
\hline 55 & $\begin{array}{c}3.1 \\
8\end{array}$ & $\begin{array}{c}2.9 \\
6\end{array}$ & $\begin{array}{c}3.1 \\
5\end{array}$ & $\begin{array}{c}3.0 \\
8\end{array}$ & $\begin{array}{c}3.0 \\
2\end{array}$ & $\begin{array}{c}3.0 \\
0\end{array}$ & $\begin{array}{c}3.0 \\
2\end{array}$ & $\begin{array}{c}2.9 \\
9\end{array}$ & 2.93 \\
\hline 60 & $\begin{array}{c}3.0 \\
8\end{array}$ & $\begin{array}{c}2.8 \\
8\end{array}$ & $\begin{array}{c}3.1 \\
0\end{array}$ & $\begin{array}{c}3.0 \\
3\end{array}$ & $\begin{array}{c}2.9 \\
9\end{array}$ & $\begin{array}{c}2.9 \\
5\end{array}$ & $\begin{array}{c}2.9 \\
8\end{array}$ & $\begin{array}{c}2.9 \\
4\end{array}$ & 2.86 \\
\hline \multicolumn{10}{|c|}{ CMC mixtures } \\
\hline
\end{tabular}


Published Online September 2019 in IJEAST (http://www.ijeast.com)

\begin{tabular}{|c|c|c|c|c|c|c|c|c|c|}
\hline $\begin{array}{c}\text { Tem } \\
\text { pera } \\
\text { ture } \\
{ }^{0} \mathrm{C}\end{array}$ & $\begin{array}{l}20 \\
\%\end{array}$ & $\begin{array}{l}15 \\
\%\end{array}$ & $\begin{array}{l}10 \\
\%\end{array}$ & $5 \%$ & $1 \%$ & $\begin{array}{c}0.5 \\
\%\end{array}$ & $\begin{array}{c}0.1 \\
\%\end{array}$ & $\begin{array}{l}0.0 \\
5 \%\end{array}$ & $\begin{array}{c}0.01 \\
\%\end{array}$ \\
\hline 30 & $\begin{array}{c}3.3 \\
3\end{array}$ & $\begin{array}{c}3.1 \\
7\end{array}$ & $\begin{array}{c}3.1 \\
0\end{array}$ & $\begin{array}{c}3.0 \\
7\end{array}$ & $\begin{array}{c}2.9 \\
4\end{array}$ & $\begin{array}{c}3.0 \\
0\end{array}$ & $\begin{array}{c}2.9 \\
1\end{array}$ & $\begin{array}{c}2.9 \\
6\end{array}$ & 2.90 \\
\hline 35 & $\begin{array}{c}3.3 \\
0\end{array}$ & $\begin{array}{c}3.1 \\
1\end{array}$ & $\begin{array}{c}3.0 \\
4\end{array}$ & $\begin{array}{c}3.0 \\
3\end{array}$ & $\begin{array}{c}2.8 \\
8\end{array}$ & $\begin{array}{c}2.9 \\
3\end{array}$ & $\begin{array}{c}2.9 \\
0\end{array}$ & $\begin{array}{c}2.9 \\
1\end{array}$ & 2.86 \\
\hline 40 & $\begin{array}{c}3.2 \\
4\end{array}$ & $\begin{array}{c}3.0 \\
2\end{array}$ & $\begin{array}{c}3.0 \\
0\end{array}$ & $\begin{array}{c}2.9 \\
8\end{array}$ & $\begin{array}{c}2.8 \\
4\end{array}$ & $\begin{array}{c}2.8 \\
4\end{array}$ & $\begin{array}{c}2.8 \\
6\end{array}$ & $\begin{array}{c}2.8 \\
0\end{array}$ & 2.81 \\
\hline 45 & $\begin{array}{c}3.2 \\
0\end{array}$ & $\begin{array}{c}2.8 \\
8\end{array}$ & $\begin{array}{c}2.9 \\
8\end{array}$ & $\begin{array}{c}2.9 \\
2\end{array}$ & $\begin{array}{c}2.8 \\
0\end{array}$ & $\begin{array}{c}2.7 \\
7\end{array}$ & $\begin{array}{c}2.8 \\
3\end{array}$ & $\begin{array}{c}2.7 \\
3\end{array}$ & 2.79 \\
\hline 50 & $\begin{array}{c}3.1 \\
8\end{array}$ & $\begin{array}{c}2.8 \\
3\end{array}$ & $\begin{array}{c}2.9 \\
4\end{array}$ & $\begin{array}{c}2.8 \\
6\end{array}$ & $\begin{array}{c}2.7 \\
8\end{array}$ & $\begin{array}{c}2.6 \\
1\end{array}$ & $\begin{array}{c}2.8 \\
0\end{array}$ & $\begin{array}{c}2.5 \\
5\end{array}$ & 2.77 \\
\hline 55 & $\begin{array}{c}3.1 \\
1\end{array}$ & $\begin{array}{c}2.6 \\
9\end{array}$ & $\begin{array}{c}2.9 \\
0\end{array}$ & $\begin{array}{c}2.8 \\
1\end{array}$ & $\begin{array}{c}2.7 \\
5\end{array}$ & $\begin{array}{c}2.5 \\
0\end{array}$ & $\begin{array}{c}2.7 \\
6\end{array}$ & $\begin{array}{c}2.4 \\
9\end{array}$ & 2.72 \\
\hline 60 & $\begin{array}{c}3.0 \\
8\end{array}$ & $\begin{array}{c}2.6 \\
4\end{array}$ & $\begin{array}{c}2.8 \\
9\end{array}$ & $\begin{array}{c}2.7 \\
7\end{array}$ & $\begin{array}{c}2.7 \\
2\end{array}$ & $\begin{array}{c}2.4 \\
6\end{array}$ & $\begin{array}{c}2.7 \\
4\end{array}$ & $\begin{array}{c}2.4 \\
0\end{array}$ & 2.69 \\
\hline & & & & $C \boldsymbol{n}$ & tures & & & & \\
\hline $\begin{array}{c}\text { Tem } \\
\text { pera } \\
\text { ture } \\
{ }^{0} \mathrm{C}\end{array}$ & $\begin{array}{l}20 \\
\%\end{array}$ & $\begin{array}{l}15 \\
\%\end{array}$ & $\begin{array}{l}10 \\
\%\end{array}$ & $5 \%$ & $1 \%$ & $\begin{array}{c}0.5 \\
\%\end{array}$ & $\begin{array}{c}0.1 \\
\%\end{array}$ & $\begin{array}{l}0.0 \\
5 \%\end{array}$ & $\begin{array}{c}0.01 \\
\%\end{array}$ \\
\hline 30 & $\begin{array}{c}2.9 \\
0\end{array}$ & $\begin{array}{c}2.9 \\
1\end{array}$ & $\begin{array}{c}2.8 \\
6\end{array}$ & $\begin{array}{c}2.8 \\
9\end{array}$ & $\begin{array}{c}2.8 \\
6\end{array}$ & $\begin{array}{c}2.9 \\
5\end{array}$ & $\begin{array}{c}2.8 \\
5\end{array}$ & $\begin{array}{c}2.9 \\
3\end{array}$ & 2.85 \\
\hline 35 & $\begin{array}{c}2.8 \\
8\end{array}$ & $\begin{array}{c}2.8 \\
6\end{array}$ & $\begin{array}{c}2.8 \\
2\end{array}$ & $\begin{array}{c}2.8 \\
3\end{array}$ & $\begin{array}{c}2.8 \\
3\end{array}$ & $\begin{array}{c}2.9 \\
0\end{array}$ & $\begin{array}{c}2.8 \\
3\end{array}$ & $\begin{array}{c}2.8 \\
8\end{array}$ & 2.82 \\
\hline 40 & $\begin{array}{c}2.8 \\
4\end{array}$ & $\begin{array}{c}2.8 \\
4\end{array}$ & $\begin{array}{c}2.7 \\
5\end{array}$ & $\begin{array}{c}2.7 \\
5\end{array}$ & $\begin{array}{c}2.7 \\
6\end{array}$ & $\begin{array}{c}2.8 \\
1\end{array}$ & $\begin{array}{c}2.7 \\
7\end{array}$ & $\begin{array}{c}2.7 \\
7\end{array}$ & 2.76 \\
\hline 45 & $\begin{array}{c}2.8 \\
0\end{array}$ & $\begin{array}{c}2.7 \\
4\end{array}$ & $\begin{array}{c}2.7 \\
3\end{array}$ & $\begin{array}{c}2.6 \\
6\end{array}$ & $\begin{array}{c}2.7 \\
5\end{array}$ & $\begin{array}{c}2.6 \\
6\end{array}$ & $\begin{array}{c}2.7 \\
4\end{array}$ & $\begin{array}{c}2.6 \\
3\end{array}$ & 2.75 \\
\hline 50 & $\begin{array}{c}2.7 \\
6\end{array}$ & $\begin{array}{c}2.6 \\
6\end{array}$ & $\begin{array}{c}2.7 \\
2\end{array}$ & $\begin{array}{c}2.5 \\
1\end{array}$ & $\begin{array}{c}2.7 \\
4\end{array}$ & $\begin{array}{c}2.6 \\
3\end{array}$ & $\begin{array}{c}2.7 \\
3\end{array}$ & $\begin{array}{c}2.6 \\
0\end{array}$ & 2.73 \\
\hline 55 & $\begin{array}{c}2.7 \\
3\end{array}$ & $\begin{array}{c}2.6 \\
1\end{array}$ & $\begin{array}{c}2.7 \\
0\end{array}$ & $\begin{array}{c}2.4 \\
8\end{array}$ & $\begin{array}{c}2.7 \\
1\end{array}$ & $\begin{array}{c}2.5 \\
9\end{array}$ & $\begin{array}{c}2.7 \\
1\end{array}$ & $\begin{array}{c}2.5 \\
4\end{array}$ & 2.70 \\
\hline 60 & $\begin{array}{c}2.7 \\
1\end{array}$ & $\begin{array}{c}2.5 \\
3\end{array}$ & $\begin{array}{c}2.6 \\
8\end{array}$ & $\begin{array}{c}2.3 \\
5\end{array}$ & $\begin{array}{c}2.6 \\
9\end{array}$ & $\begin{array}{c}2.5 \\
5\end{array}$ & $\begin{array}{c}2.6 \\
9\end{array}$ & $\begin{array}{c}2.4 \\
9\end{array}$ & 2.68 \\
\hline \multicolumn{10}{|c|}{ HPMC mixtures } \\
\hline $\begin{array}{c}\text { Tem } \\
\text { pera } \\
\text { ture } \\
{ }^{0} \mathrm{C}\end{array}$ & $\begin{array}{l}20 \\
\%\end{array}$ & $\begin{array}{l}15 \\
\%\end{array}$ & $\begin{array}{l}10 \\
\%\end{array}$ & $5 \%$ & $1 \%$ & $\begin{array}{c}0.5 \\
\%\end{array}$ & $\begin{array}{c}0.1 \\
\%\end{array}$ & $\begin{array}{l}0.0 \\
5 \%\end{array}$ & $\begin{array}{c}0.01 \\
\%\end{array}$ \\
\hline 30 & $\begin{array}{c}3.1 \\
5\end{array}$ & $\begin{array}{c}3.1 \\
2\end{array}$ & $\begin{array}{c}3.1 \\
5\end{array}$ & $\begin{array}{c}3.1 \\
6\end{array}$ & $\begin{array}{c}3.1 \\
1\end{array}$ & $\begin{array}{c}3.1 \\
0\end{array}$ & $\begin{array}{c}3.1 \\
2\end{array}$ & $\begin{array}{c}3.1 \\
5\end{array}$ & 3.10 \\
\hline 35 & $\begin{array}{c}3.1 \\
0\end{array}$ & $\begin{array}{c}3.0 \\
8\end{array}$ & $\begin{array}{c}3.1 \\
0\end{array}$ & $\begin{array}{c}3.1 \\
1\end{array}$ & $\begin{array}{c}3.1 \\
0\end{array}$ & $\begin{array}{c}3.0 \\
8\end{array}$ & $\begin{array}{c}3.1 \\
1\end{array}$ & $\begin{array}{c}3.1 \\
4\end{array}$ & 3.09 \\
\hline 40 & $\begin{array}{c}3.0 \\
8\end{array}$ & $\begin{array}{c}3.0 \\
7\end{array}$ & $\begin{array}{c}3.0 \\
6\end{array}$ & $\begin{array}{c}3.0 \\
9\end{array}$ & $\begin{array}{c}3.0 \\
8\end{array}$ & $\begin{array}{c}3.0 \\
4\end{array}$ & $\begin{array}{c}3.0 \\
7\end{array}$ & $\begin{array}{c}3.1 \\
0\end{array}$ & 3.06 \\
\hline 45 & $\begin{array}{c}3.0 \\
5\end{array}$ & $\begin{array}{c}3.0 \\
6\end{array}$ & $\begin{array}{c}3.0 \\
5\end{array}$ & $\begin{array}{c}3.0 \\
5\end{array}$ & $\begin{array}{c}3.0 \\
6\end{array}$ & $\begin{array}{c}3.0 \\
0\end{array}$ & $\begin{array}{c}3.0 \\
5\end{array}$ & $\begin{array}{c}3.0 \\
8\end{array}$ & 3.05 \\
\hline
\end{tabular}

\begin{tabular}{|c|c|c|c|c|c|c|c|c|c|}
\hline 50 & $\begin{array}{c}3.0 \\
2\end{array}$ & $\begin{array}{c}3.0 \\
3\end{array}$ & $\begin{array}{c}3.0 \\
4\end{array}$ & $\begin{array}{c}3.0 \\
3\end{array}$ & $\begin{array}{c}3.0 \\
5\end{array}$ & $\begin{array}{c}2.9 \\
9\end{array}$ & $\begin{array}{c}3.0 \\
2\end{array}$ & $\begin{array}{c}3.0 \\
4\end{array}$ & 3.07 \\
\hline 55 & $\begin{array}{c}3.0 \\
1\end{array}$ & $\begin{array}{c}3.0 \\
0\end{array}$ & $\begin{array}{c}3.0 \\
3\end{array}$ & $\begin{array}{c}3.0 \\
0\end{array}$ & $\begin{array}{c}3.0 \\
3\end{array}$ & $\begin{array}{c}2.9 \\
5\end{array}$ & $\begin{array}{c}3.0 \\
1\end{array}$ & $\begin{array}{c}3.0 \\
0\end{array}$ & 3.03 \\
\hline $\mathbf{6 0}$ & $\begin{array}{c}3.0 \\
0\end{array}$ & $\begin{array}{c}2.9 \\
7\end{array}$ & $\begin{array}{c}3.0 \\
2\end{array}$ & $\begin{array}{c}2.9 \\
7\end{array}$ & $\begin{array}{c}3.0 \\
2\end{array}$ & $\begin{array}{c}2.9 \\
3\end{array}$ & $\begin{array}{c}3.0 \\
0\end{array}$ & $\begin{array}{c}2.9 \\
3\end{array}$ & 2.99 \\
\hline
\end{tabular}

6. c.) Effect of temperature on $\mathrm{pH}$ of $\mathrm{XG}$ and GT mixtures containing $\mathrm{Hg}$ metal ion

\begin{tabular}{|c|c|c|c|c|c|c|c|c|c|}
\hline \multicolumn{10}{|c|}{$X G$ mixtures } \\
\hline $\begin{array}{c}\text { Tem } \\
\text { pera } \\
\text { ture } \\
{ }^{0} \mathrm{C}\end{array}$ & $\begin{array}{l}20 \\
\%\end{array}$ & $\begin{array}{l}15 \\
\%\end{array}$ & $\begin{array}{l}10 \\
\%\end{array}$ & $5 \%$ & $1 \%$ & $\begin{array}{c}0.5 \\
\%\end{array}$ & $\begin{array}{c}0.1 \\
\%\end{array}$ & $\begin{array}{l}0.0 \\
5 \%\end{array}$ & $\begin{array}{c}0.01 \\
\%\end{array}$ \\
\hline 30 & $\begin{array}{c}2.9 \\
1\end{array}$ & $\begin{array}{c}3.0 \\
3\end{array}$ & $\begin{array}{c}2.9 \\
1\end{array}$ & $\begin{array}{c}2.9 \\
8\end{array}$ & $\begin{array}{c}3.0 \\
7\end{array}$ & $\begin{array}{c}3.1 \\
0\end{array}$ & $\begin{array}{c}3.0 \\
5\end{array}$ & $\begin{array}{c}2.9 \\
8\end{array}$ & 2.81 \\
\hline 35 & $\begin{array}{c}2.8 \\
6\end{array}$ & $\begin{array}{c}3.0 \\
1\end{array}$ & $\begin{array}{c}2.8 \\
8\end{array}$ & $\begin{array}{c}2.9 \\
5\end{array}$ & $\begin{array}{c}3.0 \\
3\end{array}$ & $\begin{array}{c}3.0 \\
7\end{array}$ & $\begin{array}{c}3.0 \\
1\end{array}$ & $\begin{array}{c}2.9 \\
1\end{array}$ & 2.78 \\
\hline 40 & $\begin{array}{c}2.7 \\
7\end{array}$ & $\begin{array}{c}2.9 \\
6\end{array}$ & $\begin{array}{c}2.7 \\
7\end{array}$ & $\begin{array}{c}2.9 \\
0\end{array}$ & $\begin{array}{c}2.9 \\
8\end{array}$ & $\begin{array}{c}3.0 \\
5\end{array}$ & $\begin{array}{c}2.9 \\
6\end{array}$ & $\begin{array}{c}2.8 \\
5\end{array}$ & 2.73 \\
\hline 45 & $\begin{array}{c}2.6 \\
1\end{array}$ & $\begin{array}{c}2.9 \\
2\end{array}$ & $\begin{array}{c}2.6 \\
4\end{array}$ & $\begin{array}{c}2.8 \\
8\end{array}$ & $\begin{array}{c}2.9 \\
1\end{array}$ & $\begin{array}{c}3.0 \\
4\end{array}$ & $\begin{array}{c}2.9 \\
0\end{array}$ & $\begin{array}{c}2.7 \\
7\end{array}$ & 2.66 \\
\hline 50 & $\begin{array}{c}2.5 \\
3\end{array}$ & $\begin{array}{c}2.8 \\
8\end{array}$ & $\begin{array}{c}2.5 \\
9\end{array}$ & $\begin{array}{c}2.8 \\
4\end{array}$ & $\begin{array}{c}2.8 \\
7\end{array}$ & $\begin{array}{c}3.0 \\
0\end{array}$ & $\begin{array}{c}2.8 \\
6\end{array}$ & $\begin{array}{c}2.6 \\
3\end{array}$ & 2.62 \\
\hline 55 & $\begin{array}{c}2.4 \\
7\end{array}$ & $\begin{array}{c}2.8 \\
3\end{array}$ & $\begin{array}{c}2.5 \\
3\end{array}$ & $\begin{array}{c}2.7 \\
9\end{array}$ & $\begin{array}{c}2.8 \\
3\end{array}$ & $\begin{array}{c}2.9 \\
6\end{array}$ & $\begin{array}{c}2.8 \\
4\end{array}$ & $\begin{array}{c}2.5 \\
5\end{array}$ & 2.57 \\
\hline 60 & $\begin{array}{c}2.4 \\
0\end{array}$ & $\begin{array}{c}2.7 \\
7\end{array}$ & $\begin{array}{c}2.4 \\
0\end{array}$ & $\begin{array}{c}2.7 \\
5\end{array}$ & $\begin{array}{c}2.6 \\
9\end{array}$ & $\begin{array}{c}2.9 \\
4\end{array}$ & $\begin{array}{c}2.7 \\
7\end{array}$ & $\begin{array}{c}2.4 \\
2\end{array}$ & 2.55 \\
\hline \multicolumn{10}{|c|}{ GT mixtures } \\
\hline $\begin{array}{c}\text { Tem } \\
\text { pera } \\
\text { ture } \\
{ }^{0} \mathrm{C}\end{array}$ & $\begin{array}{l}20 \\
\%\end{array}$ & $\begin{array}{l}15 \\
\%\end{array}$ & $\begin{array}{l}10 \\
\%\end{array}$ & $5 \%$ & $1 \%$ & $\begin{array}{c}0.5 \\
\%\end{array}$ & $\begin{array}{c}0.1 \\
\%\end{array}$ & $\begin{array}{l}0.0 \\
5 \%\end{array}$ & $\begin{array}{c}0.01 \\
\%\end{array}$ \\
\hline 30 & $\begin{array}{c}3.0 \\
8\end{array}$ & $\begin{array}{c}3.0 \\
7\end{array}$ & $\begin{array}{c}3.1 \\
0\end{array}$ & $\begin{array}{c}2.7 \\
9\end{array}$ & $\begin{array}{c}2.9 \\
8\end{array}$ & $\begin{array}{c}2.8 \\
8\end{array}$ & $\begin{array}{c}2.9 \\
4\end{array}$ & $\begin{array}{c}3.0 \\
8\end{array}$ & 2.82 \\
\hline 35 & $\begin{array}{c}2.9 \\
6\end{array}$ & $\begin{array}{c}3.0 \\
3\end{array}$ & $\begin{array}{c}3.0 \\
2\end{array}$ & $\begin{array}{c}2.7 \\
4\end{array}$ & $\begin{array}{c}2.8 \\
4\end{array}$ & $\begin{array}{c}2.8 \\
5\end{array}$ & $\begin{array}{c}2.8 \\
8\end{array}$ & $\begin{array}{c}3.0 \\
3\end{array}$ & 2.78 \\
\hline 40 & $\begin{array}{c}2.8 \\
4\end{array}$ & $\begin{array}{c}2.9 \\
9\end{array}$ & $\begin{array}{c}2.9 \\
8\end{array}$ & $\begin{array}{c}2.7 \\
0\end{array}$ & $\begin{array}{c}2.7 \\
7\end{array}$ & $\begin{array}{c}2.7 \\
7\end{array}$ & $\begin{array}{c}2.8 \\
0\end{array}$ & $\begin{array}{c}3.0 \\
0\end{array}$ & 2.62 \\
\hline 45 & $\begin{array}{c}2.7 \\
9\end{array}$ & $\begin{array}{c}2.9 \\
1\end{array}$ & $\begin{array}{c}2.8 \\
6\end{array}$ & $\begin{array}{c}2.6 \\
5\end{array}$ & $\begin{array}{c}2.7 \\
4\end{array}$ & $\begin{array}{c}2.7 \\
6\end{array}$ & $\begin{array}{c}2.7 \\
8\end{array}$ & $\begin{array}{c}2.9 \\
7\end{array}$ & 2.56 \\
\hline 50 & $\begin{array}{c}2.6 \\
2\end{array}$ & $\begin{array}{c}2.8 \\
6\end{array}$ & $\begin{array}{c}2.7 \\
2\end{array}$ & $\begin{array}{c}2.6 \\
1\end{array}$ & $\begin{array}{c}2.6 \\
8\end{array}$ & $\begin{array}{c}2.7 \\
2\end{array}$ & $\begin{array}{c}2.6 \\
2\end{array}$ & $\begin{array}{c}2.9 \\
4\end{array}$ & 2.48 \\
\hline 55 & $\begin{array}{c}2.6 \\
0\end{array}$ & $\begin{array}{c}2.8 \\
1\end{array}$ & $\begin{array}{c}2.6 \\
2\end{array}$ & $\begin{array}{c}2.5 \\
5\end{array}$ & $\begin{array}{c}2.6 \\
6\end{array}$ & $\begin{array}{c}2.6 \\
9\end{array}$ & $\begin{array}{c}2.5 \\
0\end{array}$ & $\begin{array}{c}2.8 \\
6\end{array}$ & 2.45 \\
\hline 60 & $\begin{array}{c}2.5 \\
4\end{array}$ & $\begin{array}{c}2.7 \\
7\end{array}$ & $\begin{array}{c}2.5 \\
8\end{array}$ & $\begin{array}{c}2.4 \\
9\end{array}$ & $\begin{array}{c}2.5 \\
2\end{array}$ & $\begin{array}{c}2.6 \\
7\end{array}$ & $\begin{array}{c}2.4 \\
8\end{array}$ & $\begin{array}{c}2.7 \\
8\end{array}$ & 2.33 \\
\hline
\end{tabular}




\section{International Journal of Engineering Applied Sciences and Technology, 2019 \\ Vol. 4, Issue 5, ISSN No. 2455-2143, Pages 285-301 \\ Published Online September 2019 in IJEAST (http://www.ijeast.com)}

\subsubsection{Effect of temperature on density}

Density changes with temperature because volume changes with temperature. As temperature increases, the volume usually increases because the faster moving molecules are further apart. Thus increasing the volume decreases the density. Change in density values of $\mathrm{Fe}$ and $\mathrm{Hg}$ blank solutions with increasing temperature are given below in Fig. $3 \& 4$ respectively. The temperature effect on density values for various concentrations of biopolymer mixtures containing $\mathrm{Fe}$ metal ion are given in Fig.3.a, 3.b, 3.c, 3.d, 3.e, 3.f, 3.g, 3.h for GG, XG, Ac.G, GT, $\mathrm{Na}$ Alg., CMC, MC, HPMC mixtures respectively. Similarly effect of temperature on density values of XG-Hg, GT-Hg mixtures are given in Fig.4.a, 4.b respectively. The density curves of $\mathrm{Fe}$ and $\mathrm{Hg}$ blank (Fig.3, 4)) taken as reference curve. Density curves of all biopolymer mixtures containing different metal ions when compared with corresponding reference curve, higher density values were obtained.

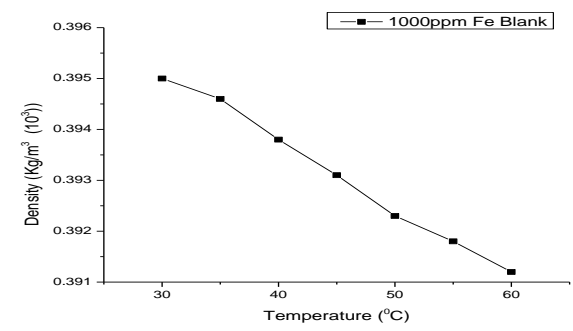

Fig.3 Effect of temperature on density of Fe blank

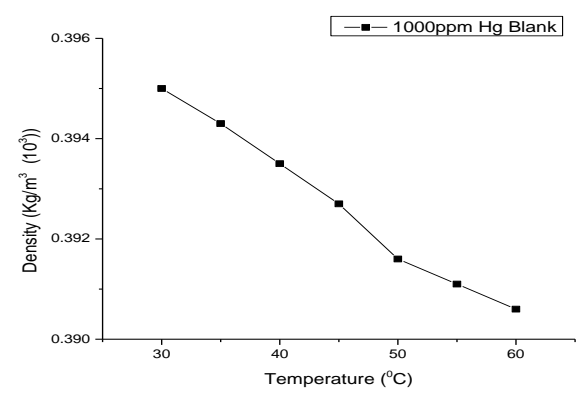

Fig.4 Effect of temperature on density of Hg blank

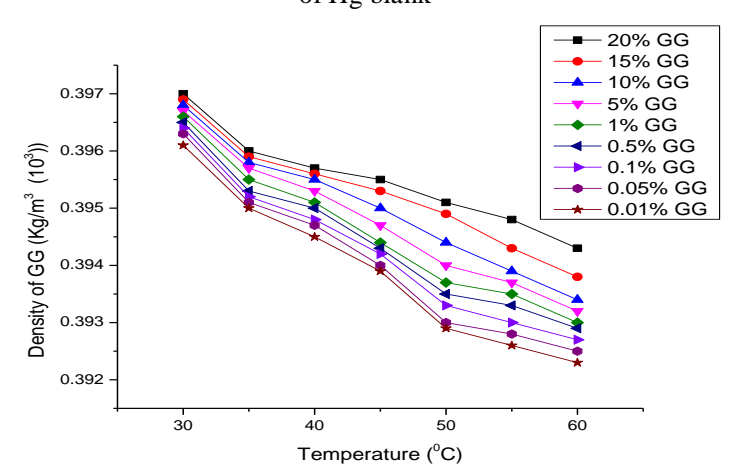

Fig.3.a Effect of temperature on density of GG-Fe mixture

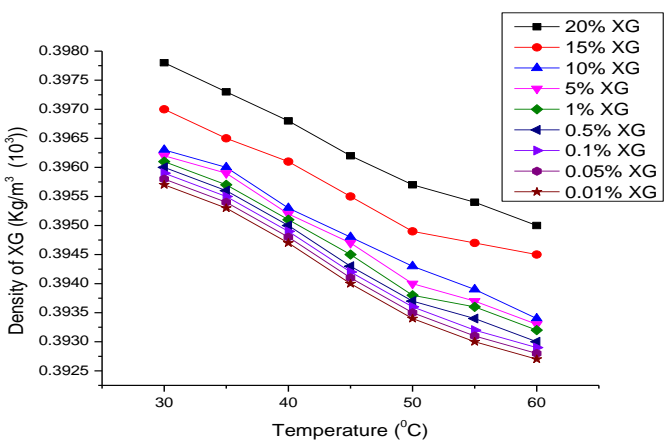

Fig.3.b Effect of temperature on density of $\mathrm{XG}-\mathrm{Fe}$ mixture

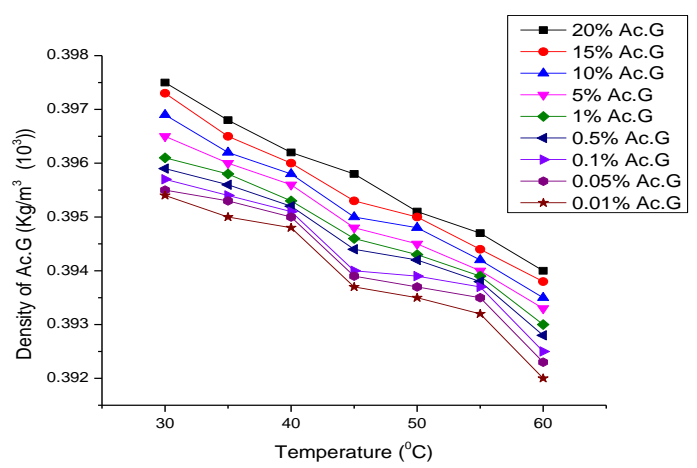

Fig.3.c Effect of temperature on density of Ac.G-Fe mixture

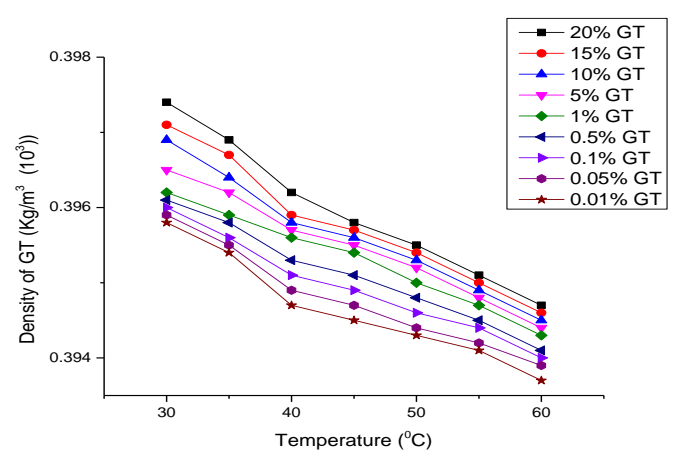

Fig.3.d Effect of temperature on density of GT-Fe mixture

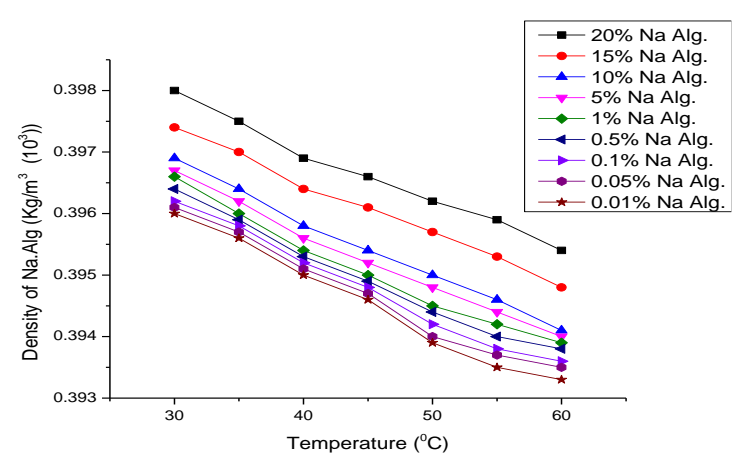

Fig.3.e Effect of temperature on density of $\mathrm{Na} \mathrm{Alg}$.-Fe mixture 


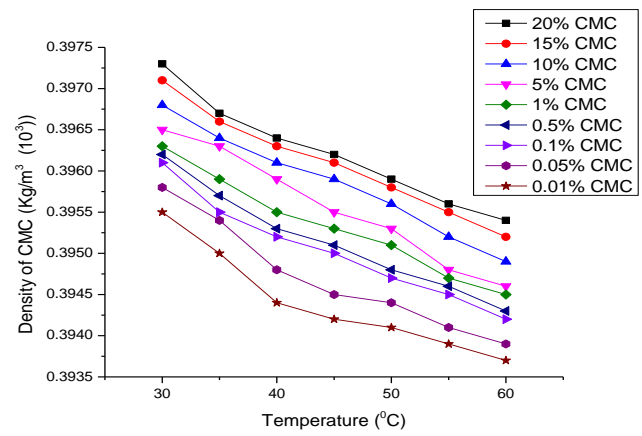

Fig.3.f Effect of temperature on density of CMC-Fe mixture

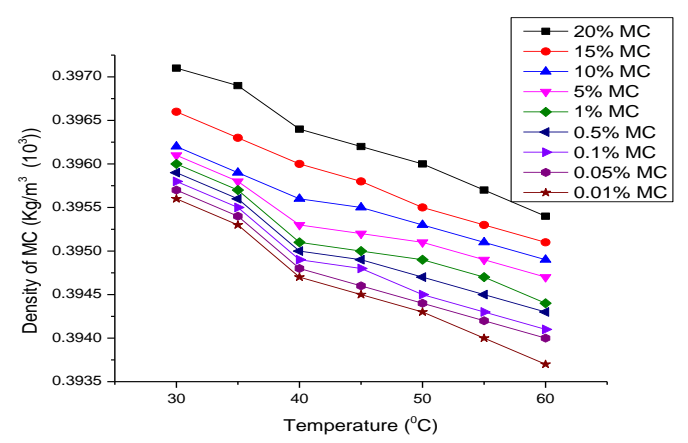

Fig.3.g Effect of temperature on density of MC-Fe mixture

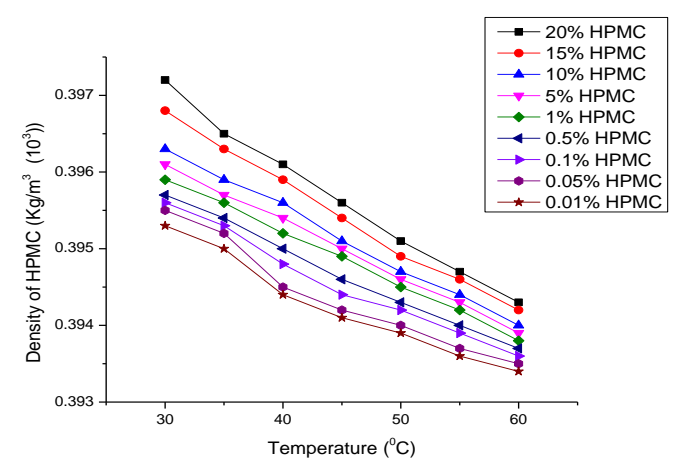

Fig.3.h Effect of temperature on density of HPMC-Fe mixtures

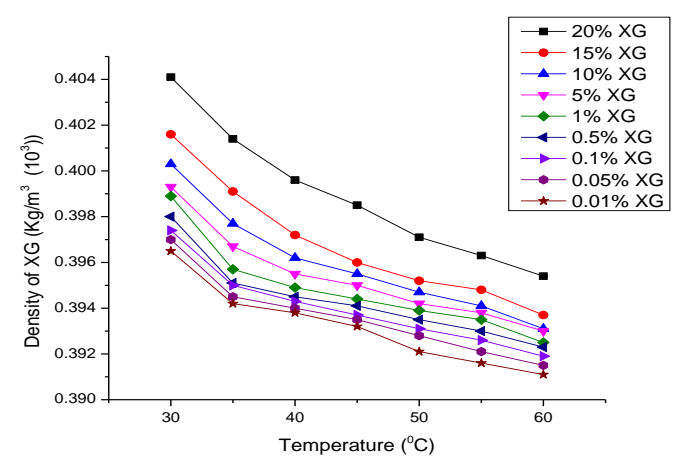

Fig.4.a Effect of temperature on density of XG-Hg mixture

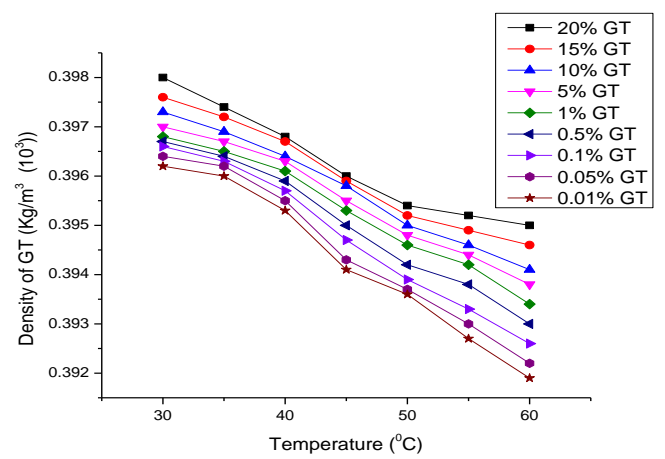

Fig.4.b Effect of temperature on density of GT-Hg mixture

As we move from higher to lower concentrations of the mixture, mass of the substance decreases density values become less. Density values of $20 \%$ and $15 \%$ mixtures of Xanthan gum-Fe (Fig.3.b), Sodium Alginate-Fe (Fig.3.e) and Methyl Cellulose-Fe (Fig.3.g) are very high. These biopolymers when mixed with Fe metal ion solution, form very thick mixture and Sodium alginate mixture becomes turbid too. Hence respective density curves lies at the top. All concentrations of Guar gum-Fe mixtures (Fig.3.a) are thick. Their initial density will be high. Later as temperature increases (after $35^{\circ} \mathrm{C}$ ) density slightly decreases. Acacia gum-Fe (Fig.3.c) and Gum tragacanth-Fe (Fig.3.d) mixtures dissolve quickly with Fe metal ion solution. Hence they do not form compact solution. All the mixtures of Ac.G-Fe and GT-Fe showed good difference in density values. CMC-Fe (Fig.3.f) mixtures also become little bit thick. But all these mixtures exhibit good density differences. In case of Fig.3.h, all lines are close to each other this is because of negligible difference in density values between different concentrations of Hydroxypropyl methyl cellulose mixtures.

At higher concentrations Xanthan gum usually becomes thick when it mixes with Mercury metal ion solution. Therefore such mixture $20 \%, 15 \%$ and $10 \%$ of XG-Hg (Fig.4.a) will give large density values. After $10 \%$ (5\% XG-Fe - 0.01\% XG-Fe), density values found to be closer since these mixtures will not become that much thick. All GT (20\% to $0.01 \%)$ mixtures are quickly soluble with Mercury metal ion solution. Also solution will not become thick. So differences between the density values are very less.

Sorption kinetics of change in density with respect to temperature also favors the absorption of metal ions from waste water.

\subsubsection{Effect of temperature on viscosity}

The viscosity of liquids decreases with increase in temperature. The cohesive force between molecules of liquids decreases. At high temperature these molecules have high energy and overcome strong cohesive forces and move freely. Therefore viscosity of liquids decreases with increase in temperature. Change in viscosity values with temperature for 
$\mathrm{Fe}$ and $\mathrm{Hg}$ blank are given below in Fig. 5 \& 6 respectively. Fig.5.a, 5.b, 5.c, 5.d, 5.e, 5.f, 5.g and 5.h represents the temperature effect on viscosity values for different Guar gum, Xanthan gum, Acacia gum, Gum tragacanth, Sodium alginate, $\mathrm{CMC}, \mathrm{MC}$ and HPMC mixtures respectively which contain $\mathrm{Fe}$ metal ion. Similarly Fig.6.a and 6.b represents temperature effect on viscosity of XG-Hg and GT-Hg mixtures respectively. Fig.5, 6 considered as reference curve. Viscosity curves of all biopolymer mixtures containing different metal ions when compared with corresponding reference curve, higher viscosity values were obtained.

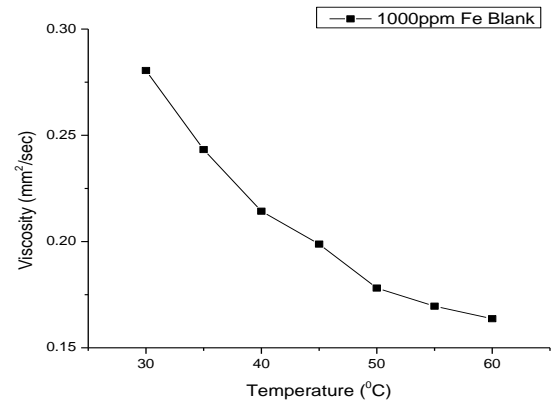

Fig.5 Effect of temperature on viscosity of Fe blank.

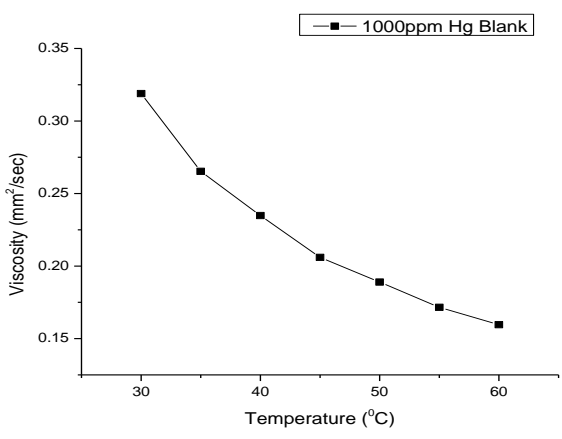

Fig.6 Temperature effect on Viscosity of $\mathrm{Hg}$ blank

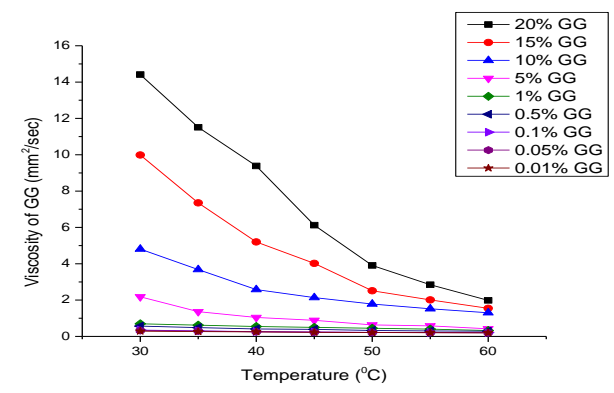

Fig.5.a Effect of temperature on viscosity of GG-Fe mixtures

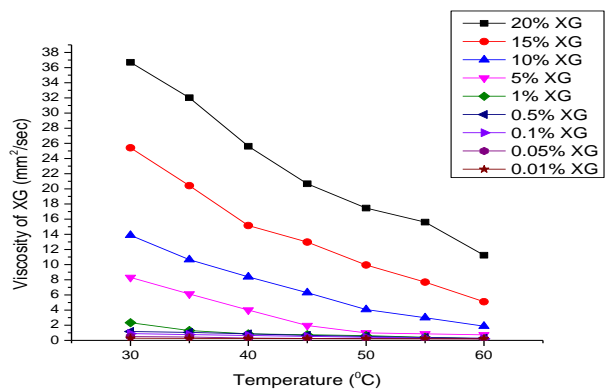

Fig.5.b Temperature effect on Viscosity of XG-Fe mixtures

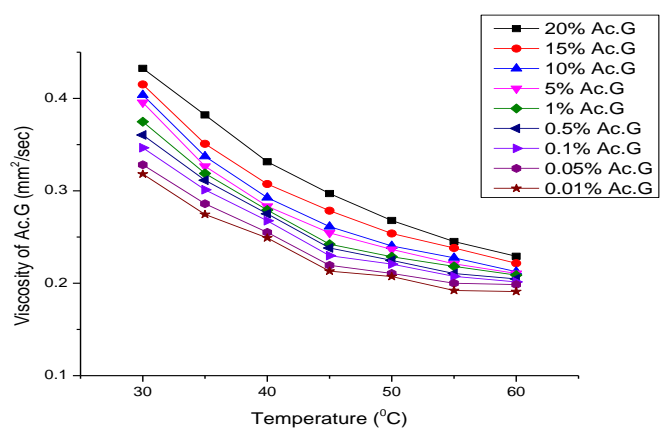

Fig.5.c Effect of temperature on viscosity of Ac.G-Fe mixtures

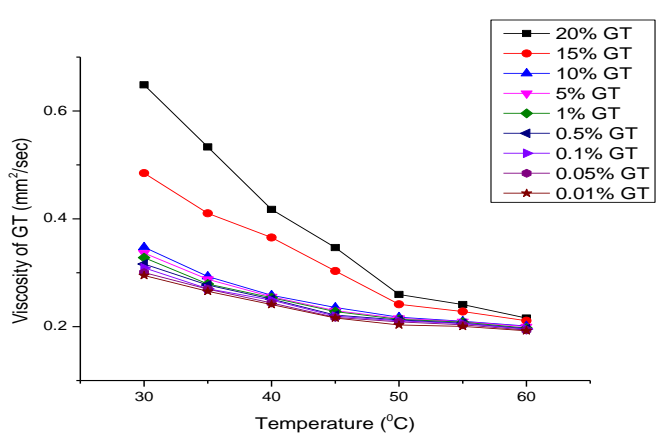

Fig.5.d Effect of temperature on viscosity of GT-Fe mixtures

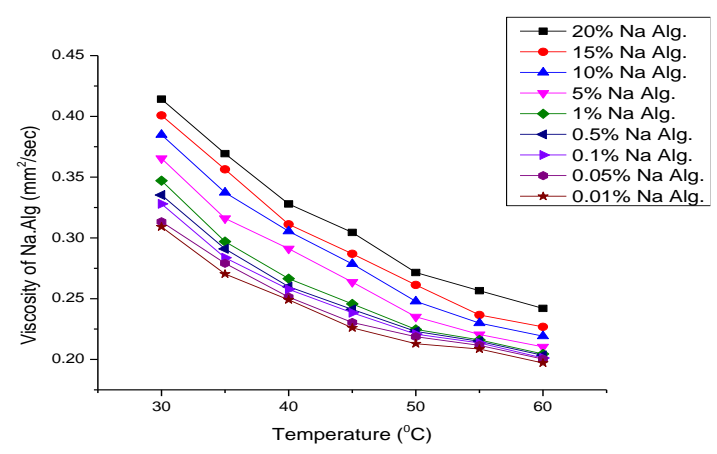

Fig.5.e Effect of temperature on viscosity of $\mathrm{Na} \mathrm{Alg.-Fe} \mathrm{mixtures}$ 


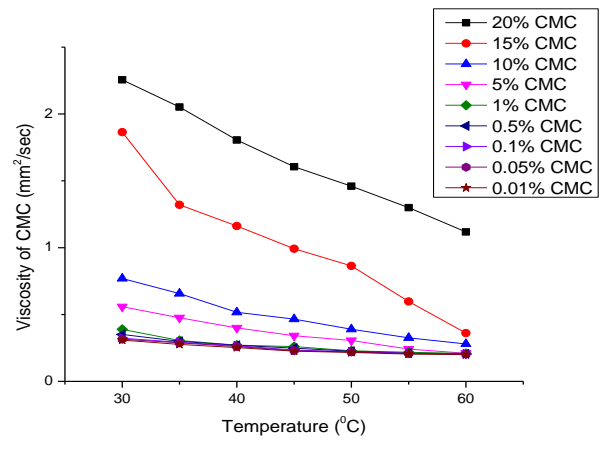

Fig.5.f Effect of temperature on viscosity of CMC-Fe mixtures

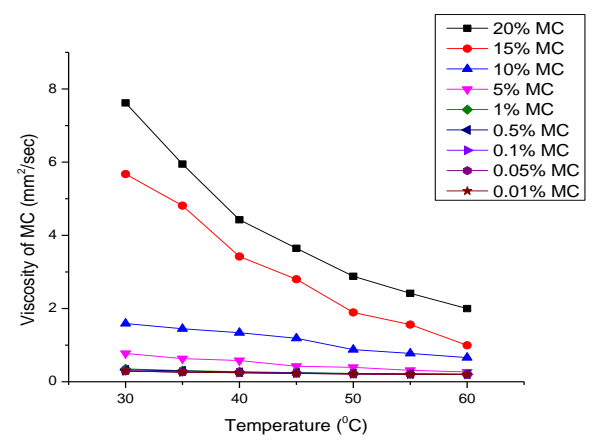

Fig.5.g Effect of temperature on viscosity of MC-Fe mixtures

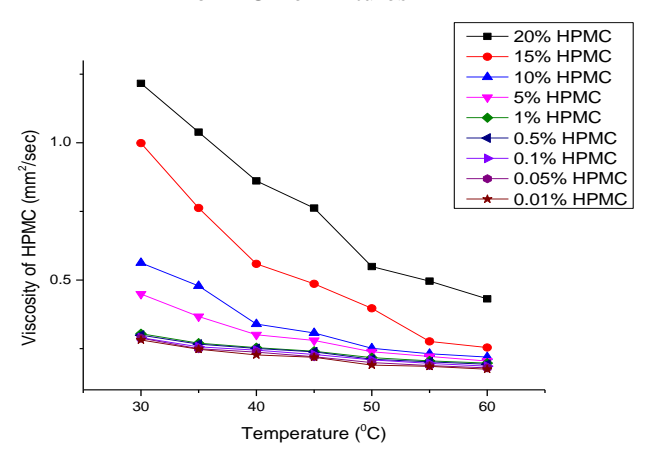

Fig.5.h Effect of temperature on viscosity of HPMC-Fe mixtures

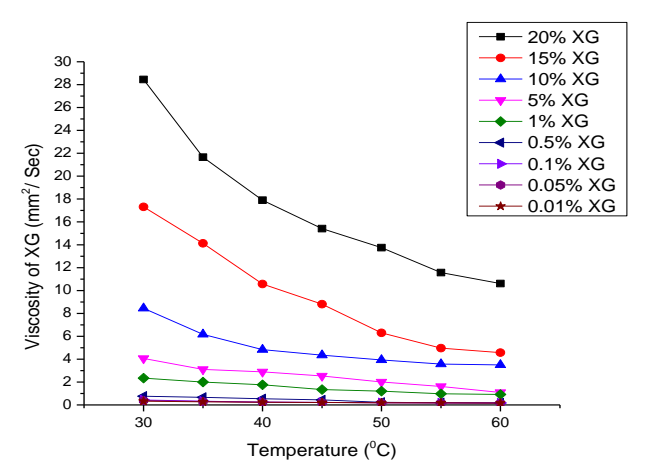

Fig.6.a Effect of temperature on viscosity of $\mathrm{XG}-\mathrm{Hg}$ mixtures

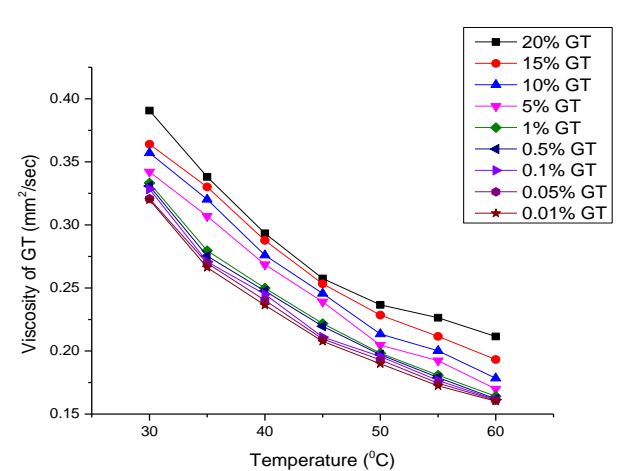

Fig.6.b Effect of temperature on viscosity of GT-Hg mixtures

Out of all biopolymer thickness of Xanthan gum-Fe (Fig.5.b) and Xanthan gum-Hg (Fig.6.a) mixtures are very high. Its viscosity values also higher than other. Thickness remains high till $10 \% \mathrm{XG}$ whereas after $10 \%$, becomes less thick also viscosity curves of $1 \%-0.01 \%$ XG mixtures of both metal ions seem to be very close or overlapping. Mixtures which become too thick when mixed with Fe metal ion solution will give higher viscosity values. Respective viscosity curves lies at the top. Such observation we can see at $20 \%$ and $15 \% \mathrm{Fe}$ metal ion mixtures of Guar gum (Fig.5.a), Gum Tragacanth (Fig.5.d), CMC (Fig.5.f), MC (Fig.5.g) and HPMC (Fig.5.h). As concentration of mixture decreases they lose their stiffness. Hence least concentrated mixtures almost occupy the same line in the curve. Viscosity values of all Acasia Gum-Fe (Fig.5.c) and Sodium alginate-Fe (Fig.3.e) mixtures showed good difference throughout. In Fig.6.b all GT-Hg mixtures (20\% to $0.01 \%$ ) are quickly soluble with $\mathrm{Hg}$ metal ion solution also it will not become thick. Hence their viscosity curves lies very close to each other, also $1 \%$ to $0.1 \%$ mixtures seem to be overlapping.

We observed that minimal amounts of $1 \%$ to $0.01 \%$ polymer mixtures are easy to handle for effective absorbance of $\mathrm{Fe}$ and $\mathrm{Hg}$ metal ions from industrial waste water. They dissolve quickly with metal ion solution. Thickness is also less.

\subsubsection{Effect of temperature on Ultrasonic sound velocity}

Sorption kinetics of ultrasonic sound velocity carried out as a part of solution property studies of biopolymers with heavy metal ions. Temperature is also a condition that affects speed of sound [13]. Molecules at higher temperature have more energy thus they can vibrate faster. In this experiment, velocity of ultrasonic sound decreases as temperature rises. Ultrasonic sound velocity of blank $\mathrm{Fe}$ and $\mathrm{Hg}$ solution at different temperatures and effect of temperature on ultrasonic sound velocity of various biopolymer mixtures with corresponding metal ion are given below (Table 7). All these values decreased with increasing temperature but no characteristic changes observed between the values of ultrasonic sound velocity for different concentrations of biopolymer mixtures. 
International Journal of Engineering Applied Sciences and Technology, 2019

Vol. 4, Issue 5, ISSN No. 2455-2143, Pages 285-301

Published Online September 2019 in IJEAST (http://www.ijeast.com)

Table 7. a.) Effect of temperature on ultrasonic sound velocity of Fe,

\begin{tabular}{|c|c|c|}
\hline $\begin{array}{c}\text { Temperature } \\
\left({ }^{\boldsymbol{0}} \boldsymbol{C}\right)\end{array}$ & $\begin{array}{c}\text { He blank solutions } \\
\text { Velocity } \text { of Fe } \\
\text { blank }(\boldsymbol{m} / \mathbf{s})\end{array}$ & $\begin{array}{c}\text { Ultrasonic } \\
\text { Velocity of Ho } \\
\text { blank }(\boldsymbol{m} / \mathbf{s})\end{array}$ \\
\hline $\mathbf{3 0}$ & 1609 & 1605 \\
\hline $\mathbf{3 5}$ & 1603 & 1602 \\
\hline $\mathbf{4 0}$ & 1600 & 1600 \\
\hline $\mathbf{4 5}$ & 1595 & 1593 \\
\hline $\mathbf{5 0}$ & 1574 & 1589 \\
\hline $\mathbf{5 5}$ & 1561 & 1576 \\
\hline $\mathbf{6 0}$ & 1543 & 1557 \\
\hline
\end{tabular}

7. b.) Effect of temperature on ultrasonic sound velocity of biopolymer mixtures contain Fe metal ion

\begin{tabular}{|c|c|c|c|c|c|c|c|c|c|}
\hline \multicolumn{10}{|c|}{ GG mixtures $(\mathrm{m} / \mathrm{s})$} \\
\hline $\begin{array}{c}\text { Tem } \\
\text { perat } \\
\text { ure } \\
{ }^{\circ} \mathrm{C}\end{array}$ & $\begin{array}{l}20 \\
\%\end{array}$ & $\begin{array}{l}15 \\
\%\end{array}$ & $\begin{array}{l}10 \\
\%\end{array}$ & $5 \%$ & $1 \%$ & $\begin{array}{c}0.5 \\
\%\end{array}$ & $\begin{array}{c}0.1 \\
\%\end{array}$ & $\begin{array}{l}0.0 \\
5 \%\end{array}$ & $\begin{array}{c}0.01 \\
\%\end{array}$ \\
\hline 30 & $\begin{array}{c}172 \\
4\end{array}$ & $\begin{array}{l}16 \\
55\end{array}$ & $\begin{array}{l}16 \\
46\end{array}$ & $\begin{array}{l}16 \\
12\end{array}$ & $\begin{array}{l}17 \\
08\end{array}$ & $\begin{array}{l}16 \\
88\end{array}$ & $\begin{array}{l}16 \\
30\end{array}$ & $\begin{array}{l}16 \\
61\end{array}$ & 1645 \\
\hline 35 & $\begin{array}{c}165 \\
7\end{array}$ & $\begin{array}{l}16 \\
23\end{array}$ & $\begin{array}{l}16 \\
38\end{array}$ & $\begin{array}{l}16 \\
09\end{array}$ & $\begin{array}{l}16 \\
89\end{array}$ & $\begin{array}{l}16 \\
59\end{array}$ & $\begin{array}{l}16 \\
14\end{array}$ & $\begin{array}{l}16 \\
38\end{array}$ & 1622 \\
\hline 40 & $\begin{array}{c}160 \\
6\end{array}$ & $\begin{array}{l}16 \\
11\end{array}$ & $\begin{array}{l}16 \\
19\end{array}$ & $\begin{array}{l}15 \\
88\end{array}$ & $\begin{array}{l}16 \\
55\end{array}$ & $\begin{array}{l}16 \\
33\end{array}$ & $\begin{array}{l}16 \\
01\end{array}$ & $\begin{array}{l}16 \\
15\end{array}$ & 1608 \\
\hline 45 & $\begin{array}{c}159 \\
1\end{array}$ & $\begin{array}{l}15 \\
96\end{array}$ & $\begin{array}{l}15 \\
93\end{array}$ & $\begin{array}{l}15 \\
70\end{array}$ & $\begin{array}{l}16 \\
43\end{array}$ & $\begin{array}{l}16 \\
11\end{array}$ & $\begin{array}{l}15 \\
86\end{array}$ & $\begin{array}{l}15 \\
93\end{array}$ & 1580 \\
\hline 50 & $\begin{array}{c}158 \\
6\end{array}$ & $\begin{array}{l}15 \\
63\end{array}$ & $\begin{array}{l}15 \\
77\end{array}$ & $\begin{array}{l}15 \\
57\end{array}$ & $\begin{array}{l}16 \\
18\end{array}$ & $\begin{array}{l}15 \\
99\end{array}$ & $\begin{array}{l}15 \\
70\end{array}$ & $\begin{array}{l}15 \\
78\end{array}$ & 1566 \\
\hline 55 & $\begin{array}{c}157 \\
0\end{array}$ & $\begin{array}{l}15 \\
41\end{array}$ & $\begin{array}{l}15 \\
46\end{array}$ & $\begin{array}{l}15 \\
46\end{array}$ & $\begin{array}{l}15 \\
82\end{array}$ & $\begin{array}{l}15 \\
74\end{array}$ & $\begin{array}{l}15 \\
59\end{array}$ & $\begin{array}{l}15 \\
63\end{array}$ & 1545 \\
\hline 60 & $\begin{array}{c}153 \\
6\end{array}$ & $\begin{array}{l}15 \\
16\end{array}$ & $\begin{array}{l}15 \\
31\end{array}$ & $\begin{array}{l}15 \\
22\end{array}$ & $\begin{array}{l}15 \\
63\end{array}$ & $\begin{array}{l}15 \\
55\end{array}$ & $\begin{array}{l}15 \\
41\end{array}$ & $\begin{array}{l}15 \\
54\end{array}$ & 1521 \\
\hline \multicolumn{10}{|c|}{$X G$ mixtures $(\mathrm{m} / \mathrm{s})$} \\
\hline $\begin{array}{c}\text { Tem } \\
\text { perat } \\
\text { ure } \\
{ }^{0} \mathrm{C}\end{array}$ & $\begin{array}{l}20 \\
\%\end{array}$ & $\begin{array}{l}15 \\
\%\end{array}$ & $\begin{array}{l}10 \\
\%\end{array}$ & $5 \%$ & $1 \%$ & $\begin{array}{c}0.5 \\
\%\end{array}$ & $\begin{array}{c}0.1 \\
\%\end{array}$ & $\begin{array}{l}0.0 \\
5 \%\end{array}$ & $\begin{array}{c}0.01 \\
\%\end{array}$ \\
\hline 30 & $\begin{array}{c}160 \\
9\end{array}$ & $\begin{array}{l}15 \\
88\end{array}$ & $\begin{array}{l}16 \\
07\end{array}$ & $\begin{array}{l}16 \\
13\end{array}$ & $\begin{array}{l}15 \\
94\end{array}$ & $\begin{array}{l}15 \\
95\end{array}$ & $\begin{array}{l}16 \\
04\end{array}$ & $\begin{array}{l}16 \\
11\end{array}$ & 1616 \\
\hline 35 & $\begin{array}{c}160 \\
3\end{array}$ & $\begin{array}{l}15 \\
83\end{array}$ & $\begin{array}{l}16 \\
01\end{array}$ & $\begin{array}{l}16 \\
02\end{array}$ & $\begin{array}{l}15 \\
90\end{array}$ & $\begin{array}{l}15 \\
86\end{array}$ & $\begin{array}{l}15 \\
98\end{array}$ & $\begin{array}{l}16 \\
05\end{array}$ & 1608 \\
\hline 40 & $\begin{array}{c}160 \\
0\end{array}$ & $\begin{array}{l}15 \\
76\end{array}$ & $\begin{array}{l}15 \\
93\end{array}$ & $\begin{array}{l}15 \\
93\end{array}$ & $\begin{array}{l}15 \\
82\end{array}$ & $\begin{array}{l}15 \\
71\end{array}$ & $\begin{array}{l}15 \\
90\end{array}$ & $\begin{array}{l}15 \\
97\end{array}$ & 1591 \\
\hline 45 & $\begin{array}{c}159 \\
7\end{array}$ & $\begin{array}{l}15 \\
69\end{array}$ & $\begin{array}{l}15 \\
86\end{array}$ & $\begin{array}{l}15 \\
72\end{array}$ & $\begin{array}{l}15 \\
60\end{array}$ & $\begin{array}{l}15 \\
63\end{array}$ & $\begin{array}{l}15 \\
79\end{array}$ & $\begin{array}{l}15 \\
84\end{array}$ & 1583 \\
\hline 50 & $\begin{array}{c}159 \\
1\end{array}$ & $\begin{array}{l}15 \\
50\end{array}$ & $\begin{array}{l}15 \\
63\end{array}$ & $\begin{array}{l}15 \\
66\end{array}$ & $\begin{array}{l}15 \\
48\end{array}$ & $\begin{array}{l}15 \\
48\end{array}$ & $\begin{array}{l}15 \\
71\end{array}$ & $\begin{array}{l}15 \\
72\end{array}$ & 1568 \\
\hline 55 & $\begin{array}{c}158 \\
4\end{array}$ & $\begin{array}{l}15 \\
47\end{array}$ & $\begin{array}{l}15 \\
55\end{array}$ & $\begin{array}{l}15 \\
58\end{array}$ & $\begin{array}{l}15 \\
22\end{array}$ & $\begin{array}{l}15 \\
42\end{array}$ & $\begin{array}{l}15 \\
56\end{array}$ & $\begin{array}{l}15 \\
59\end{array}$ & 1554 \\
\hline
\end{tabular}

\begin{tabular}{|c|c|c|c|c|c|c|c|c|c|}
\hline 60 & $\begin{array}{c}157 \\
8\end{array}$ & $\begin{array}{l}15 \\
38\end{array}$ & $\begin{array}{l}15 \\
41\end{array}$ & $\begin{array}{l}15 \\
44\end{array}$ & $\begin{array}{l}15 \\
04\end{array}$ & $\begin{array}{l}15 \\
33\end{array}$ & $\begin{array}{l}15 \\
47\end{array}$ & $\begin{array}{l}15 \\
43\end{array}$ & 1539 \\
\hline \multicolumn{10}{|c|}{ Ac.G mixtures $(\mathrm{m} / \mathrm{s})$} \\
\hline $\begin{array}{c}\text { Tem } \\
\text { perat } \\
\text { ure } \\
{ }^{0} \mathrm{C}\end{array}$ & $\begin{array}{l}20 \\
\%\end{array}$ & $\begin{array}{l}15 \\
\%\end{array}$ & $\begin{array}{l}10 \\
\%\end{array}$ & $5 \%$ & $1 \%$ & $\begin{array}{c}0.5 \\
\%\end{array}$ & $\begin{array}{c}0.1 \\
\%\end{array}$ & $\begin{array}{l}0.0 \\
5 \%\end{array}$ & $\begin{array}{c}0.01 \\
\%\end{array}$ \\
\hline 30 & $\begin{array}{c}157 \\
5\end{array}$ & $\begin{array}{l}15 \\
81\end{array}$ & $\begin{array}{l}15 \\
65\end{array}$ & $\begin{array}{l}15 \\
79\end{array}$ & $\begin{array}{l}15 \\
90\end{array}$ & $\begin{array}{l}15 \\
88\end{array}$ & $\begin{array}{l}15 \\
66\end{array}$ & $\begin{array}{l}15 \\
87\end{array}$ & 1597 \\
\hline 35 & $\begin{array}{c}156 \\
4\end{array}$ & $\begin{array}{l}15 \\
77\end{array}$ & $\begin{array}{l}15 \\
55\end{array}$ & $\begin{array}{l}15 \\
63\end{array}$ & $\begin{array}{l}15 \\
73\end{array}$ & $\begin{array}{l}15 \\
74\end{array}$ & $\begin{array}{l}15 \\
58\end{array}$ & $\begin{array}{l}15 \\
79\end{array}$ & 1587 \\
\hline 40 & $\begin{array}{c}155 \\
2\end{array}$ & $\begin{array}{l}15 \\
62\end{array}$ & $\begin{array}{l}15 \\
42\end{array}$ & $\begin{array}{l}15 \\
45\end{array}$ & $\begin{array}{l}15 \\
60\end{array}$ & $\begin{array}{l}15 \\
58\end{array}$ & $\begin{array}{l}15 \\
49\end{array}$ & $\begin{array}{l}15 \\
69\end{array}$ & 1575 \\
\hline 45 & $\begin{array}{c}154 \\
8\end{array}$ & $\begin{array}{l}15 \\
48\end{array}$ & $\begin{array}{l}15 \\
30\end{array}$ & $\begin{array}{l}15 \\
38\end{array}$ & $\begin{array}{l}15 \\
48\end{array}$ & $\begin{array}{l}15 \\
49\end{array}$ & $\begin{array}{l}15 \\
26\end{array}$ & 15 & 1566 \\
\hline 50 & $\begin{array}{c}153 \\
0\end{array}$ & $\begin{array}{l}15 \\
30\end{array}$ & $\begin{array}{l}15 \\
19\end{array}$ & 15 & $\begin{array}{l}15 \\
28\end{array}$ & 15 & 15 & 15 & 1542 \\
\hline 55 & $\begin{array}{c}152 \\
2\end{array}$ & $\begin{array}{l}15 \\
22\end{array}$ & $\begin{array}{l}15 \\
08\end{array}$ & $\begin{array}{l}15 \\
06\end{array}$ & $\begin{array}{l}15 \\
06\end{array}$ & $\begin{array}{l}15 \\
15\end{array}$ & $\begin{array}{l}15 \\
09\end{array}$ & $\begin{array}{l}15 \\
25\end{array}$ & 1533 \\
\hline 60 & $\begin{array}{c}150 \\
9\end{array}$ & $\begin{array}{l}15 \\
16\end{array}$ & $\begin{array}{l}15 \\
03\end{array}$ & $\begin{array}{l}14 \\
94\end{array}$ & $\begin{array}{l}14 \\
96\end{array}$ & $\begin{array}{l}15 \\
02\end{array}$ & $\begin{array}{l}15 \\
00\end{array}$ & $\begin{array}{l}15 \\
14\end{array}$ & 1511 \\
\hline \multicolumn{10}{|c|}{ GT mixtures $(\mathrm{m} / \mathrm{s})$} \\
\hline $\begin{array}{c}\text { Tem } \\
\text { perat } \\
\text { ure } \\
{ }^{0} \mathrm{C}\end{array}$ & $\begin{array}{l}20 \\
\%\end{array}$ & $\begin{array}{l}15 \\
\%\end{array}$ & $\begin{array}{l}10 \\
\%\end{array}$ & $5 \%$ & $1 \%$ & $\begin{array}{c}0.5 \\
\%\end{array}$ & $\begin{array}{c}0.1 \\
\%\end{array}$ & $\begin{array}{l}0.0 \\
5 \%\end{array}$ & $\begin{array}{c}0.01 \\
\%\end{array}$ \\
\hline 30 & $\begin{array}{c}162 \\
1\end{array}$ & $\begin{array}{l}16 \\
45\end{array}$ & $\begin{array}{l}16 \\
30\end{array}$ & $\begin{array}{l}16 \\
16\end{array}$ & $\begin{array}{l}16 \\
36\end{array}$ & $\begin{array}{l}16 \\
04\end{array}$ & $\begin{array}{l}16 \\
39\end{array}$ & $\begin{array}{l}16 \\
26\end{array}$ & 1620 \\
\hline 35 & $\begin{array}{c}161 \\
3\end{array}$ & $\begin{array}{l}16 \\
26\end{array}$ & $\begin{array}{l}16 \\
22\end{array}$ & $\begin{array}{l}16 \\
07\end{array}$ & $\begin{array}{l}16 \\
25\end{array}$ & $\begin{array}{l}16 \\
00\end{array}$ & $\begin{array}{l}16 \\
22\end{array}$ & $\begin{array}{l}16 \\
13\end{array}$ & 1611 \\
\hline 40 & $\begin{array}{c}160 \\
8\end{array}$ & $\begin{array}{l}16 \\
18\end{array}$ & $\begin{array}{l}16 \\
14\end{array}$ & $\begin{array}{l}15 \\
95\end{array}$ & $\begin{array}{l}16 \\
18\end{array}$ & $\begin{array}{l}15 \\
98\end{array}$ & $\begin{array}{l}16 \\
10\end{array}$ & $\begin{array}{l}16 \\
04\end{array}$ & 1602 \\
\hline 45 & $\begin{array}{c}159 \\
4\end{array}$ & $\begin{array}{l}16 \\
01\end{array}$ & $\begin{array}{l}16 \\
08\end{array}$ & $\begin{array}{l}15 \\
79\end{array}$ & $\begin{array}{l}16 \\
10\end{array}$ & $\begin{array}{l}15 \\
83\end{array}$ & $\begin{array}{l}16 \\
04\end{array}$ & $\begin{array}{l}15 \\
94\end{array}$ & 1591 \\
\hline 50 & $\begin{array}{c}158 \\
7\end{array}$ & $\begin{array}{l}15 \\
88\end{array}$ & $\begin{array}{l}16 \\
01\end{array}$ & $\begin{array}{l}15 \\
56\end{array}$ & $\begin{array}{l}16 \\
02\end{array}$ & $\begin{array}{l}15 \\
71\end{array}$ & $\begin{array}{l}15 \\
91\end{array}$ & $\begin{array}{l}15 \\
84\end{array}$ & 1579 \\
\hline 55 & $\begin{array}{c}156 \\
4\end{array}$ & $\begin{array}{l}15 \\
74\end{array}$ & $\begin{array}{l}15 \\
96\end{array}$ & $\begin{array}{l}15 \\
44\end{array}$ & $\begin{array}{l}15 \\
93\end{array}$ & $\begin{array}{l}15 \\
66\end{array}$ & $\begin{array}{l}15 \\
86\end{array}$ & $\begin{array}{l}15 \\
68\end{array}$ & 1573 \\
\hline 60 & $\begin{array}{c}154 \\
5\end{array}$ & $\begin{array}{l}15 \\
60\end{array}$ & $\begin{array}{l}15 \\
83\end{array}$ & $\begin{array}{l}15 \\
36\end{array}$ & $\begin{array}{l}15 \\
89\end{array}$ & $\begin{array}{l}15 \\
59\end{array}$ & $\begin{array}{l}15 \\
77\end{array}$ & $\begin{array}{l}15 \\
50\end{array}$ & 1568 \\
\hline \multicolumn{10}{|c|}{$\mathrm{Na}$ Alg. mixtures $(\mathrm{m} / \mathrm{s})$} \\
\hline $\begin{array}{c}\text { Tem } \\
\text { perat } \\
\text { ure } \\
{ }^{0} \mathrm{C}\end{array}$ & $\begin{array}{l}20 \\
\%\end{array}$ & $\begin{array}{l}15 \\
\%\end{array}$ & $\begin{array}{l}10 \\
\%\end{array}$ & $5 \%$ & $1 \%$ & $\begin{array}{c}0.5 \\
\%\end{array}$ & $\begin{array}{c}0.1 \\
\%\end{array}$ & $\begin{array}{l}0.0 \\
5 \%\end{array}$ & $\begin{array}{c}0.01 \\
\%\end{array}$ \\
\hline 30 & $\begin{array}{c}158 \\
4\end{array}$ & $\begin{array}{l}15 \\
96\end{array}$ & $\begin{array}{l}15 \\
99\end{array}$ & $\begin{array}{l}15 \\
79\end{array}$ & $\begin{array}{l}15 \\
95\end{array}$ & $\begin{array}{l}15 \\
75\end{array}$ & $\begin{array}{l}15 \\
69\end{array}$ & $\begin{array}{l}15 \\
91\end{array}$ & 1588 \\
\hline 35 & $\begin{array}{c}156 \\
4\end{array}$ & $\begin{array}{l}15 \\
88\end{array}$ & $\begin{array}{l}15 \\
86\end{array}$ & $\begin{array}{l}15 \\
63\end{array}$ & $\begin{array}{l}15 \\
75\end{array}$ & $\begin{array}{l}15 \\
46\end{array}$ & $\begin{array}{l}15 \\
47\end{array}$ & $\begin{array}{l}15 \\
76\end{array}$ & 1568 \\
\hline
\end{tabular}




\begin{tabular}{|c|c|c|c|c|c|c|c|c|c|}
\hline & 154 & 15 & 15 & 15 & 15 & 15 & 15 & 15 & 1535 \\
\hline 40 & 7 & 67 & 66 & 42 & 44 & 23 & 36 & 64 & \\
\hline & 151 & 15 & 15 & 15 & 15 & 15 & 15 & 15 & 1514 \\
\hline 45 & 4 & 39 & 42 & 35 & 32 & 11 & 18 & 48 & \\
\hline & 149 & 15 & 15 & 15 & 15 & 15 & 15 & 15 & 1500 \\
\hline 50 & 4 & 28 & 33 & 28 & 15 & 06 & 09 & 37 & \\
\hline & 147 & 15 & 15 & 15 & 15 & 14 & 14 & 15 & 1490 \\
\hline 55 & 6 & 21 & 18 & 17 & 04 & 78 & 86 & 17 & \\
\hline & 146 & 15 & 15 & 15 & 14 & 14 & 14 & 15 & 1479 \\
\hline 60 & 6 & 09 & 07 & 06 & 86 & 64 & 73 & 01 & \\
\hline \multicolumn{10}{|c|}{ CMC mixtures $(\mathrm{m} / \mathrm{s})$} \\
\hline Tem & 20 & 15 & 10 & $5 \%$ & $1 \%$ & 0.5 & 0.1 & 0.0 & 0.01 \\
\hline $\begin{array}{c}\text { perat } \\
\text { ure } \\
{ }^{0} \mathrm{C}\end{array}$ & $\%$ & $\%$ & $\%$ & & & $\%$ & $\%$ & $5 \%$ & $\%$ \\
\hline & 160 & 15 & 16 & 16 & 16 & 15 & 15 & 16 & 1594 \\
\hline 30 & 2 & 98 & 00 & 21 & 03 & 97 & 91 & 16 & \\
\hline & 159 & 15 & 15 & 16 & 15 & 15 & 15 & 16 & 1588 \\
\hline 35 & 4 & 90 & 88 & 14 & 96 & 76 & 71 & 11 & \\
\hline & 159 & 15 & 15 & 16 & 15 & 15 & 15 & 16 & 1580 \\
\hline 40 & 1 & 84 & 79 & 04 & 88 & 49 & 62 & 04 & \\
\hline & 158 & 15 & 15 & 15 & 15 & 15 & 15 & 15 & 1577 \\
\hline 45 & 6 & 77 & 71 & 95 & 63 & 38 & 47 & 99 & \\
\hline & 157 & 15 & 15 & 15 & 15 & 15 & 15 & 15 & 1563 \\
\hline 50 & 5 & 60 & 63 & 80 & 41 & 26 & 36 & 91 & \\
\hline & 156 & 15 & 15 & 15 & 15 & 15 & 15 & 15 & 1544 \\
\hline 55 & 4 & 55 & 48 & 71 & 38 & 21 & 22 & 79 & \\
\hline & 156 & 15 & 15 & 15 & 15 & 15 & 15 & 15 & 1531 \\
\hline 60 & 0 & 43 & 35 & 62 & 26 & 17 & 18 & 62 & \\
\hline \multicolumn{10}{|c|}{ MC mixtures $(\mathrm{m} / \mathrm{s})$} \\
\hline $\begin{array}{c}\text { Tem } \\
\text { perat }\end{array}$ & 20 & 15 & 10 & $5 \%$ & $1 \%$ & 0.5 & 0.1 & $\begin{array}{l}0.0 \\
5 \%\end{array}$ & 0.01 \\
\hline $\begin{array}{c}\text { ure } \\
{ }^{0} \mathrm{C}\end{array}$ & & & & & & & & & \\
\hline & 158 & 15 & 16 & 16 & 15 & 15 & 16 & 15 & 1605 \\
\hline 30 & 6 & 93 & 01 & 03 & 85 & 90 & 07 & 87 & \\
\hline & 157 & 15 & 15 & 15 & 15 & 15 & 15 & 15 & 1599 \\
\hline 35 & 4 & 86 & 91 & 87 & 73 & 80 & 90 & 67 & \\
\hline & 156 & 15 & 15 & 15 & 15 & 15 & 15 & 15 & 1588 \\
\hline 40 & 1 & 66 & 83 & 63 & 54 & 66 & 77 & 63 & \\
\hline & 154 & 15 & 15 & 15 & 15 & 15 & 15 & 15 & 1553 \\
\hline 45 & 5 & 47 & 76 & 51 & 38 & 44 & 61 & 45 & \\
\hline & 152 & 15 & 15 & 15 & 15 & 15 & 15 & 15 & 1563 \\
\hline 50 & 8 & 33 & 66 & 43 & 26 & 25 & 49 & 38 & \\
\hline & 151 & 15 & 15 & 15 & 15 & 15 & 15 & 15 & 1558 \\
\hline 55 & 2 & 18 & 53 & 28 & 14 & 11 & 39 & 21 & \\
\hline & 150 & 15 & 15 & 15 & 15 & 15 & 15 & 15 & 1542 \\
\hline 60 & 8 & 09 & 38 & 17 & 03 & 01 & 26 & 14 & \\
\hline
\end{tabular}

\begin{tabular}{|c|c|c|c|c|c|c|c|c|c|}
\hline \multicolumn{10}{|c|}{ HPMC mixtures $(\mathrm{m} / \mathrm{s})$} \\
\hline $\begin{array}{c}\text { Tem } \\
\text { perat } \\
\text { ure } \\
{ }^{0} \mathrm{C}\end{array}$ & $\begin{array}{l}20 \\
\%\end{array}$ & $\begin{array}{l}15 \\
\%\end{array}$ & $\begin{array}{l}10 \\
\%\end{array}$ & $5 \%$ & $1 \%$ & $\begin{array}{c}0.5 \\
\%\end{array}$ & $\begin{array}{c}0.1 \\
\%\end{array}$ & $\begin{array}{l}0.0 \\
5 \%\end{array}$ & $\begin{array}{c}0.01 \\
\%\end{array}$ \\
\hline \multirow[b]{2}{*}{30} & 161 & 16 & 15 & 16 & 15 & 15 & 16 & 15 & 1601 \\
\hline & 9 & 24 & 94 & 12 & 99 & 87 & 02 & 79 & \\
\hline \multirow[b]{2}{*}{35} & 160 & 16 & 15 & 16 & 15 & 15 & 15 & 15 & 1589 \\
\hline & 6 & 12 & 63 & 04 & 86 & 75 & 90 & 70 & \\
\hline \multirow[b]{2}{*}{40} & 158 & 16 & 15 & 15 & 15 & 15 & 15 & 15 & 1569 \\
\hline & 6 & 01 & 40 & 84 & 74 & 45 & 79 & 58 & \\
\hline \multirow[b]{2}{*}{45} & 156 & 15 & 15 & 15 & 15 & 15 & 15 & 15 & 1533 \\
\hline & 9 & 85 & 31 & 75 & 58 & 21 & 62 & 45 & \\
\hline \multirow[b]{2}{*}{50} & 155 & 15 & 15 & 15 & 15 & 15 & 15 & 15 & 1520 \\
\hline & 5 & 73 & 20 & 53 & 33 & 09 & 46 & 29 & \\
\hline \multirow[b]{2}{*}{55} & 153 & 15 & 15 & 15 & 15 & 14 & 15 & 15 & 1503 \\
\hline & 8 & 59 & 19 & 48 & 10 & 99 & 22 & 11 & \\
\hline \multirow[b]{2}{*}{60} & 151 & 15 & 15 & 15 & 14 & 14 & 15 & 15 & 1488 \\
\hline & 4 & 46 & 07 & 21 & 96 & 76 & 03 & 00 & \\
\hline
\end{tabular}

7. c.) Effect of temperature on ultrasonic sound velocity of biopolymer mixtures contain $\mathrm{Hg}$ metal ion

\begin{tabular}{|c|c|c|c|c|c|c|c|c|c|}
\hline \multicolumn{10}{|c|}{$X G$ mixtures $(\mathrm{m} / \mathrm{s})$} \\
\hline $\begin{array}{c}\text { Tem } \\
\text { perat } \\
\text { ure } \\
{ }^{0} \mathrm{C}\end{array}$ & $\begin{array}{l}20 \\
\%\end{array}$ & $\begin{array}{l}15 \\
\%\end{array}$ & $\begin{array}{l}10 \\
\%\end{array}$ & $5 \%$ & $1 \%$ & $\begin{array}{c}0.5 \\
\%\end{array}$ & $\begin{array}{c}0.1 \\
\%\end{array}$ & $\begin{array}{l}0.0 \\
5 \%\end{array}$ & $\begin{array}{c}0.01 \\
\%\end{array}$ \\
\hline 30 & $\begin{array}{c}161 \\
0\end{array}$ & $\begin{array}{l}15 \\
95\end{array}$ & $\begin{array}{l}16 \\
05\end{array}$ & $\begin{array}{l}16 \\
07\end{array}$ & $\begin{array}{l}16 \\
13\end{array}$ & $\begin{array}{l}16 \\
16\end{array}$ & $\begin{array}{l}15 \\
94\end{array}$ & $\begin{array}{l}16 \\
10\end{array}$ & 1607 \\
\hline 35 & $\begin{array}{c}160 \\
3\end{array}$ & $\begin{array}{l}15 \\
89\end{array}$ & $\begin{array}{l}16 \\
04\end{array}$ & $\begin{array}{l}15 \\
84\end{array}$ & $\begin{array}{l}16 \\
07\end{array}$ & $\begin{array}{l}16 \\
00\end{array}$ & $\begin{array}{l}15 \\
93\end{array}$ & $\begin{array}{l}16 \\
05\end{array}$ & 1601 \\
\hline 40 & $\begin{array}{c}159 \\
7\end{array}$ & $\begin{array}{l}15 \\
84\end{array}$ & $\begin{array}{l}15 \\
99\end{array}$ & $\begin{array}{l}15 \\
74\end{array}$ & $\begin{array}{l}15 \\
76\end{array}$ & $\begin{array}{l}15 \\
89\end{array}$ & $\begin{array}{l}15 \\
70\end{array}$ & $\begin{array}{l}15 \\
95\end{array}$ & 1600 \\
\hline 45 & $\begin{array}{c}158 \\
6\end{array}$ & $\begin{array}{l}15 \\
75\end{array}$ & $\begin{array}{l}15 \\
96\end{array}$ & $\begin{array}{l}15 \\
67\end{array}$ & $\begin{array}{l}15 \\
43\end{array}$ & $\begin{array}{l}15 \\
76\end{array}$ & $\begin{array}{l}15 \\
65\end{array}$ & $\begin{array}{l}15 \\
82\end{array}$ & 1598 \\
\hline 50 & $\begin{array}{c}157 \\
3\end{array}$ & $\begin{array}{l}15 \\
66\end{array}$ & $\begin{array}{l}15 \\
87\end{array}$ & $\begin{array}{l}15 \\
54\end{array}$ & $\begin{array}{l}15 \\
39\end{array}$ & $\begin{array}{l}15 \\
52\end{array}$ & $\begin{array}{l}15 \\
26\end{array}$ & $\begin{array}{l}15 \\
72\end{array}$ & 1584 \\
\hline 55 & $\begin{array}{c}156 \\
0\end{array}$ & $\begin{array}{l}15 \\
48\end{array}$ & $\begin{array}{l}15 \\
62\end{array}$ & $\begin{array}{l}15 \\
43\end{array}$ & $\begin{array}{l}15 \\
26\end{array}$ & $\begin{array}{l}15 \\
43\end{array}$ & $\begin{array}{l}15 \\
08\end{array}$ & $\begin{array}{l}15 \\
68\end{array}$ & 1580 \\
\hline 60 & $\begin{array}{c}155 \\
3\end{array}$ & $\begin{array}{l}15 \\
12\end{array}$ & $\begin{array}{l}15 \\
43\end{array}$ & $\begin{array}{l}15 \\
18\end{array}$ & $\begin{array}{l}14 \\
98\end{array}$ & $\begin{array}{l}15 \\
10\end{array}$ & $\begin{array}{l}14 \\
96\end{array}$ & $\begin{array}{l}15 \\
43\end{array}$ & 1575 \\
\hline \multicolumn{10}{|c|}{ GT mixtures $(\mathrm{m} / \mathrm{s})$} \\
\hline $\begin{array}{c}\text { Tem } \\
\text { perat } \\
\text { ure } \\
{ }^{0} \mathrm{C}\end{array}$ & $\begin{array}{l}20 \\
\%\end{array}$ & $\begin{array}{l}15 \\
\%\end{array}$ & $\begin{array}{l}10 \\
\%\end{array}$ & $5 \%$ & $1 \%$ & $\begin{array}{c}0.5 \\
\%\end{array}$ & $\begin{array}{c}0.1 \\
\%\end{array}$ & $\begin{array}{l}0.0 \\
5 \%\end{array}$ & $\begin{array}{c}0.01 \\
\%\end{array}$ \\
\hline 30 & $\begin{array}{c}160 \\
7\end{array}$ & $\begin{array}{l}16 \\
04\end{array}$ & $\begin{array}{l}16 \\
03\end{array}$ & $\begin{array}{l}16 \\
00\end{array}$ & $\begin{array}{l}16 \\
05\end{array}$ & $\begin{array}{l}16 \\
08\end{array}$ & $\begin{array}{l}16 \\
04\end{array}$ & $\begin{array}{l}16 \\
02\end{array}$ & 1607 \\
\hline
\end{tabular}


Published Online September 2019 in IJEAST (http://www.ijeast.com)

\begin{tabular}{|c|c|c|c|c|c|c|c|c|c|}
\hline $\mathbf{3 5}$ & 160 & 15 & 15 & 15 & 15 & 15 & 16 & 15 & 1604 \\
& 0 & 88 & 81 & 96 & 96 & 91 & 01 & 90 & \\
\hline \multirow{2}{*}{$\mathbf{4 0}$} & 159 & 15 & 15 & 15 & 15 & 15 & 15 & 15 & 1586 \\
& 6 & 77 & 73 & 85 & 89 & 81 & 96 & 70 & \\
\hline \multirow{2}{*}{$\mathbf{4 5}$} & 158 & 15 & 15 & 15 & 15 & 15 & 15 & 15 & 1583 \\
& 9 & 63 & 61 & 63 & 79 & 76 & 72 & 63 & \\
\hline \multirow{2}{*}{$\mathbf{5 0}$} & 158 & 15 & 15 & 15 & 15 & 15 & 15 & 15 & 1561 \\
& 0 & 53 & 37 & 46 & 43 & 57 & 29 & 52 & \\
\hline \multirow{2}{*}{$\mathbf{5 5}$} & 157 & 15 & 15 & 15 & 15 & 15 & 15 & 15 & 1553 \\
& 4 & 26 & 06 & 31 & 38 & 45 & 11 & 39 & \\
\hline \multirow{2}{*}{$\mathbf{0 0}$} & 156 & 15 & 14 & 15 & 15 & 15 & 14 & 15 & 1545 \\
& & & 98 & 20 & 20 & 30 & 98 & 01 & \\
\hline
\end{tabular}

\subsection{Spectrophotometric study}

Solution containing $\mathrm{Fe}$ (II) and $\mathrm{Hg}$ (II) metal ions with different concentrations of bio-polymers analyzed using Visible Double Beam spectrophotometer. Absorption maximum obtained for different concentrations of biopolymer mixtures. Maximum absorption has obtained at $355 \mathrm{~nm}$ for all the biopolymer mixtures containing Fe metal ion as well as for blank solution of Fe. Similarly maximum absorption obtained at $491 \mathrm{~nm}$ for $\mathrm{Hg}$ blank also for its XG and GT mixtures. Absorption values, for Fe blank at $355 \mathrm{~nm}$ is 0.051 and for $\mathrm{Hg}$ blank solution at $491 \mathrm{~nm}$ is 0.504 . For every mixture, respective blank reading has taken. Spectroscopic readings for Guar gum, Xanthan gum, Acacia gum, Gum tragacanth, Sodium alginate, CMC, Methyl Cellulose and HPMC mixtures which contain Fe metal ion are shown in Fig.7.a, 7.b, 7.c, 7.d, 7.e, 7.f, 7.g and 7.h respectively. Fig.8.a and 8.b represents spectral readings for XG-Hg and GT-Hg mixtures respectively.

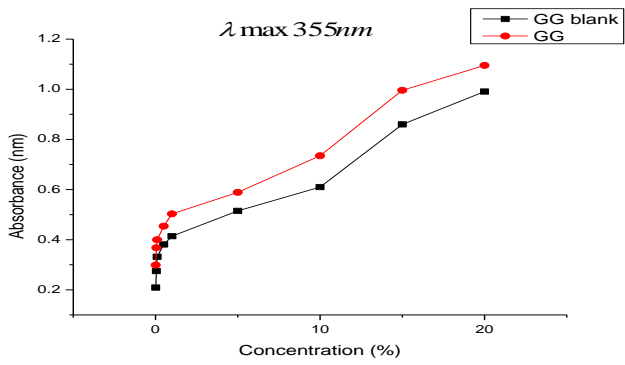

Fig.7.a Spectral readings for GG-Fe mixtures

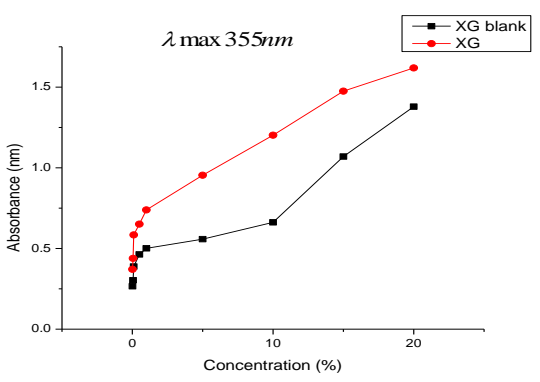

Fig.7.b Spectral readings for XG-Fe mixtures.

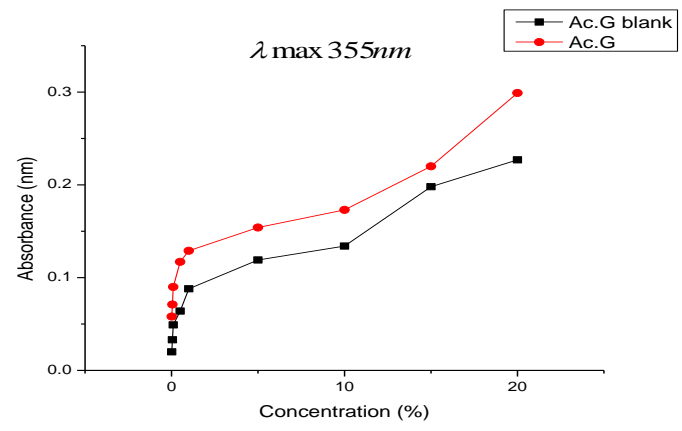

Fig.7.c Spectral readings for Ac.G-Fe mixtures

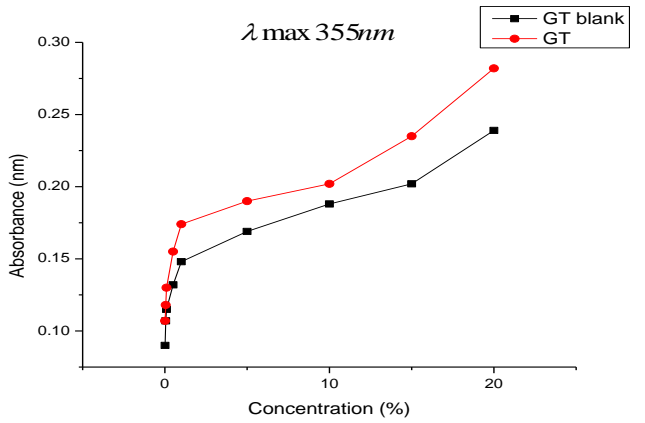

Fig.7.d Spectral readings for GT-Fe mixtures.

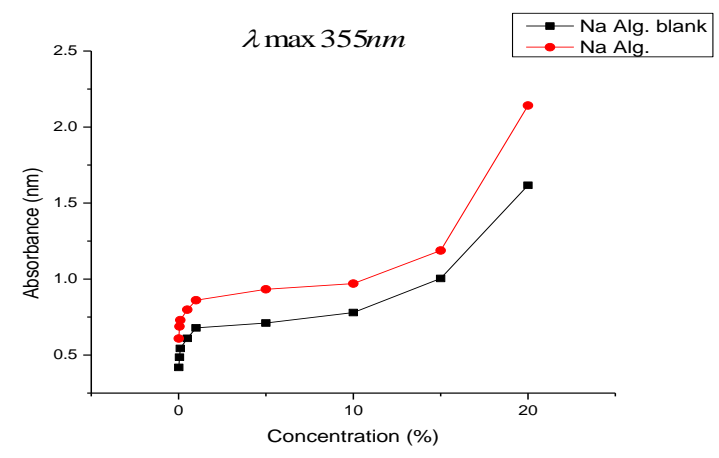

Fig.7.e Spectral readings for $\mathrm{Na}$ Alg.-Fe mixtures

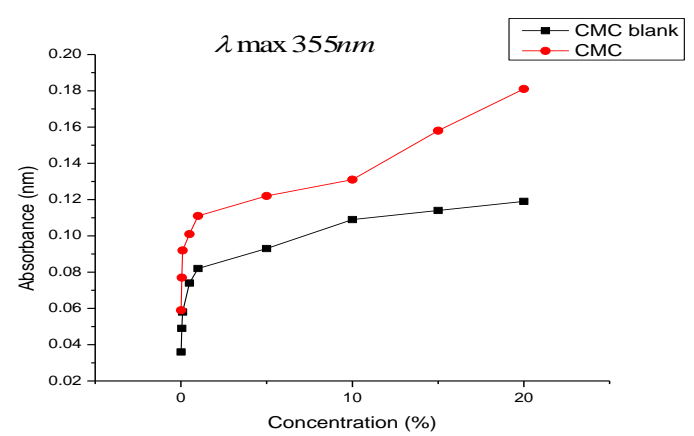

Fig.7.f Spectral readings for CMC-Fe mixtures 


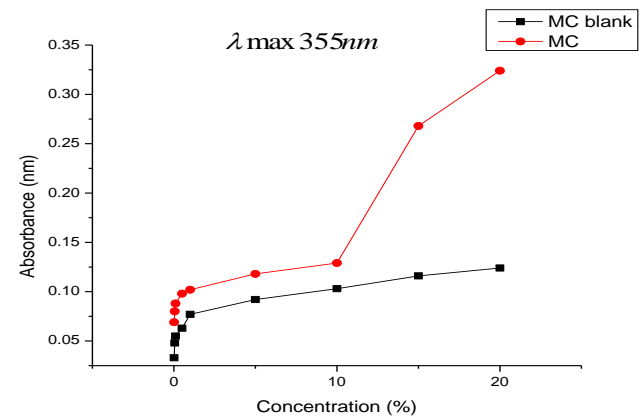

Fig.7.g Spectral readings for MC-Fe mixtures

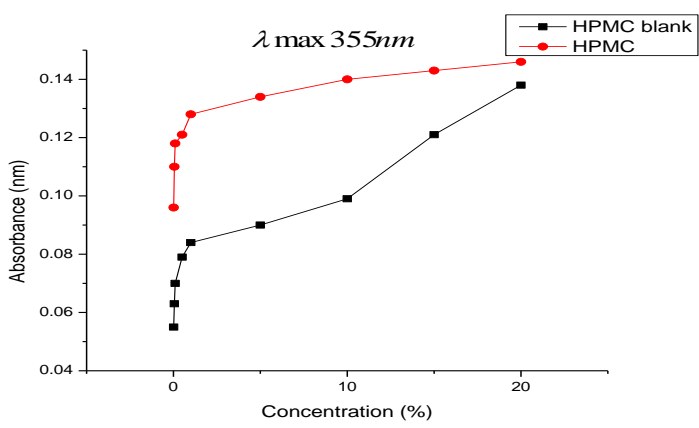

Fig.7.h Spectral readings for HPMC-Fe mixtures

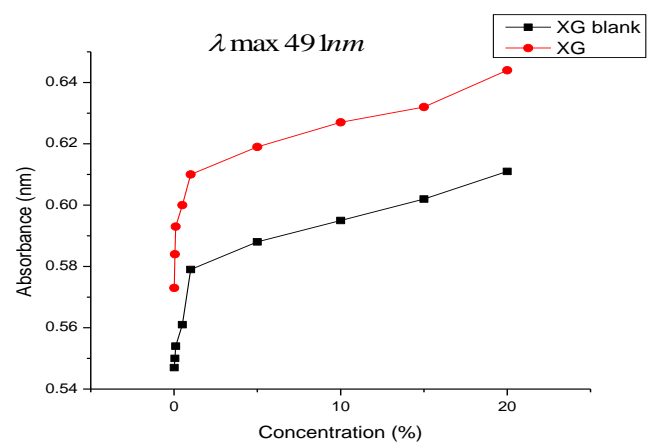

Fig.8.a Spectral readings for XG-Hg mixtures.

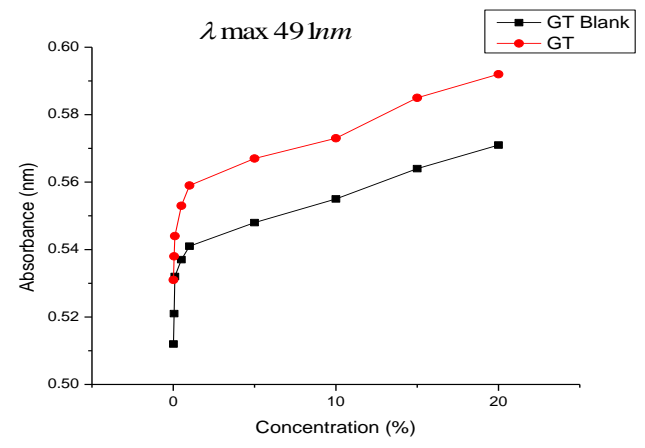

Fig.8.b Spectral readings for GT-Hg mixtures

\section{CONCLUSION}

Study has shown that biopolymers such as Guar gum, Xanthan gum, Acacia gum, Gum tragacanth, Sodium alginate, Carboxymethyl cellulose, Methyl cellulose and HPMC mixtures can absorb significant amount of Fe (II) metal ion whereas Xanthan gum and Gum tragacanth mixtures can absorb significant amount of $\mathrm{Hg}$ metal ion from industrial waste water. Minimal quantity of biopolymer mixture that is $0.01 \%$ or $0.1 \mathrm{mg}$ of biopolymer can effectively absorb Fe (II) and $\mathrm{Hg}$ (II) metal ions from waste water instead of using large quantity. Sorption kinetics of biopolymers studied at different concentrations at different temperatures. Study revealed that, solution property studies like change $\mathrm{pH}$, viscosity, density, absorption capacity and ultrasonic sound velocity with and without the sorbed metal ions, decreases with increase in temperature. Acidic $\mathrm{pH}$ range of $2-3 \mathrm{pH}$ noticed throughout in every mixture. Biopolymer mixtures which form very thick with $\mathrm{Fe}$ and $\mathrm{Hg}$ metal ion solution will have greater density and viscosity values. Remaining mixtures either close to each other or seem to be overlapping. Maximum absorption observed at $355 \mathrm{~nm}$ for all Fe related biopolymers also for Fe blank. For $\mathrm{Hg}$ blank solution, XG-Hg and GT-Hg mixtures absorption maximum obtained at $491 \mathrm{~nm}$.

\section{ACKNOWLEDGMENT}

This study is supported by Department of Chemistry and Research Centre, Sahyadri College of Engineering \& Management, Adyar. Mangalore. India. On behalf of all authors, the corresponding author states that there is no conflict of interest.

\section{REFERENCE}

[1] Bajpai S.K and Johnson S. (2005) "Superabsorbent hydrogels for removal of divalent toxic ions Part I: synthesis and swelling characterization"; Reactive and Functional Polymers. 62(3) (pp. 271-283).

[2] Barakat M A. (2011) "New trends in removing heavy metals from industrial waste water"; Arabian Journal of Chemistry. Vol. 4 (pp 361-377).

[3] Forstner U. (1983) Metal pollution in the aquatic environment. 2nd edition. New York: Springer- Verlag; 18(2). pp. 486.

[4] Kasgoz H, Ozgumus S, Orbay M. (2003) "Modified polyacrylamide hydrogels and their application in removal of heavy metal ions"; Polymer. Vol. 44 (pp 1785-1793)

[5] Niu H, Volesky B (2003) "Characteristics of anionic metal species biosorption with waste crab shells"; Hydrometallurgy. 71 (pp 209-215).

[6] Palanisamy K, Nomanbhay S M. (2005) "Removal of heavy metal from industrial waste water using chitosan coated oil palm shell charcoal"; Electronic Journal of Biotechnology. 8(1) (pp 43-53).

[7] Robb B, Lennox B. (2011) "The electrospinning process, conditions and control"; Electrospinning for Tissue Regeneration. (pp 51-66). 
[8] Sahin Y, Ozturk A. (2005) "Biosorpion of chromium (VI) ions from aqueous solution by the bacterium Bacillus thuringiensis"; Process Biochemistry. Vol 40 (pp 18951901).

[9] Thivaios I, Bokias G. (2010) “Adsorption of nile red by poly(N-isopropylacrylamide) gels in binary water/ tetrahydrofuran mixtures"; Journal of Applied Polymer Science. 116 (pp 1509-1514).

[10] Van de Velde K, Kiekens P. (2002) "Biopolymers: Overview of several properties and consequences on their applications"; Polymer Testing. Vol. 21 (pp 433-442)

[11] Volesky B. (1990) Biosorption of heavy metals. Florida: CRC Press.

[12] Volesky B. (2001) "Detoxification of metal-bearing effluents, Biosorption for the next century"; Hydrometallurgy. Vol.59 (pp 203-216)

[13] Yadawa P K. (2016) "Effect of temperature dependence ultrasonic velocities and attenuation of GaP nanowires"; Journal of Theoretical and Applied Physics. Vol.10 (pp 203-209). 